\title{
SARS-CoV-2, COVID-19 and the aging immune system
}

\author{
Juliet M. Bartleson', Dina Radenkovic ${ }^{2,3}$, Anthony J. Covarrubias ${ }^{4,5}$, David Furman ${ }^{1,6}$, \\ Daniel A. Winer ${ }^{1,6 \bowtie}$ and Eric Verdin ${ }^{1,6 凶}$
}

The coronavirus disease 2019 (COVID-19) pandemic is a global health threat with particular risk for severe disease and death in older adults and in adults with age-related metabolic and cardiovascular disease. Recent advances in the science of aging have highlighted how aging pathways control not only lifespan but also healthspan - the healthy years of life. Here, we discuss the aging immune system and its ability to respond to severe acute respiratory syndrome coronavirus 2 (SARS-CoV-2). We specifically focus on the intersect of severe COVID-19 and immunosenescence to highlight pathways that may be determinant for the risk of complications and death following infection with SARS-CoV-2. New or adapted therapeutics that target agingassociated pathways may be important tools to reduce the burden of death and long-term disability caused by this pandemic. Proposed interventions aimed at immunosenescence could enhance immune function not only in older adults but in susceptible younger individuals as well, ultimately improving complications of severe COVID-19 for all ages.

$\mathrm{T}$ he immune system changes with age in nearly every aspect, generally resulting in a decline in pathogen immunity with increased age. This diminished capacity of the aged immune system is clinically evident, as aging is associated with high morbidity and mortality rates for various infections and significant reductions in vaccine efficacy ${ }^{1-6}$. Thus, as the emergent SARSCoV-2 coronavirus began to circulate the globe early in 2020, it was reasonable to expect that the older population might be especially susceptible to poorer outcomes of COVID-19, the disease caused by SARS-CoV-2. Indeed, data from Wuhan, China showed that age was the primary risk factor associated with COVID-19 progression to acute respiratory distress syndrome (ARDS) and end-organ failure $^{7}$, which has since been corroborated by many others ${ }^{8-10}$.

COVID-19 has taken a devastating toll on the entire population, but particularly older adults. As of 24 May 2021, the Centers for Disease Control and Prevention reported nearly 590,000 total deaths from COVID-19 in the United States alone, with an estimated $80 \%$ of those deaths occurring in individuals aged 65 years or older $^{11,12}$. Compared to a 5-17-year-old reference group, the rate of hospitalization and death due to COVID-19 is approximately 1,300 times higher in individuals between the age of 65 and 74 years and 8,700 times higher in individuals 85 years and older in the United States ${ }^{11}$. While aged individuals have a higher prevalence of comorbidities that are also independently associated with increased risk of severe COVID-19, including cardiovascular disease, diabetes, chronic obstructive pulmonary disease, chronic kidney disease, cancer and others ${ }^{10}$, chronological age is still the single greatest risk factor for COVID-19 mortality ${ }^{13}$.

Thus, this review will focus on the intersect between aging and detrimental SARS-CoV-2 host-pathogen interactions during severe COVID-19 and detail physiological and immunological mechanisms underpinning both circumstances. We will also highlight targets for new or adapted therapeutics that may improve aspects of immunosenescence implicated in these shared pathways. Directed enhancement of the aging immune system may subsequently reduce the burden of death and long-term disability caused by COVID-19.

Aging, inflammaging and immunosenescence: an overview As humans age, the presence of systemic basal inflammatory mediators increases independently of acute immune challenges in a phenomenon known as inflammaging. This persistent, lowgrade, chronic inflammation has been speculated to drive many chronic diseases associated with aging and is also a main contributor to immunosenescence, a term defined as overall changes to the immune system as we age, including a reduced ability to fight new infections ${ }^{14}$. It is generally accepted that inflammaging occurs in response to an accumulation of exogenous and endogenous physiological stresses over time and is primarily mediated by immune cells

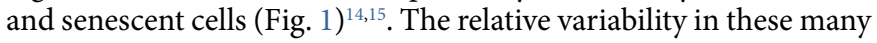
stressors from person to person is likely to explain why humans have wide variances in their biological inflammatory age across set chronological age time points ${ }^{15,16}$. This is important to consider when studying older adults, as those aged $\geq 65$ years old are not a monolithic group.

Immune cells produce many of the inflammatory mediators found in aging tissues, which can also impact their own function. The increased presence of pro-inflammatory cytokines or danger signals leads to constitutive low-level engagement of immune cell signaling events such as those from Janus kinase (JAK)-signal transducer and activator of transcription (STAT), MyD88, nuclear

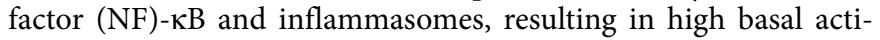
vation but impaired immune cell responses to further cytokine and pattern-recognition receptor (PRR) stimulation ${ }^{17,18}$. Thus, many immune cell subsets become hyporesponsive to acute challenges as we age ${ }^{17,19-25}$. This may help to explain why mediators of systemic chronic inflammation have been linked to suboptimal vaccine responses not only in older adults ${ }^{26,27}$ but in younger individuals as well ${ }^{28}$. Immunosenescence is further characterized by 


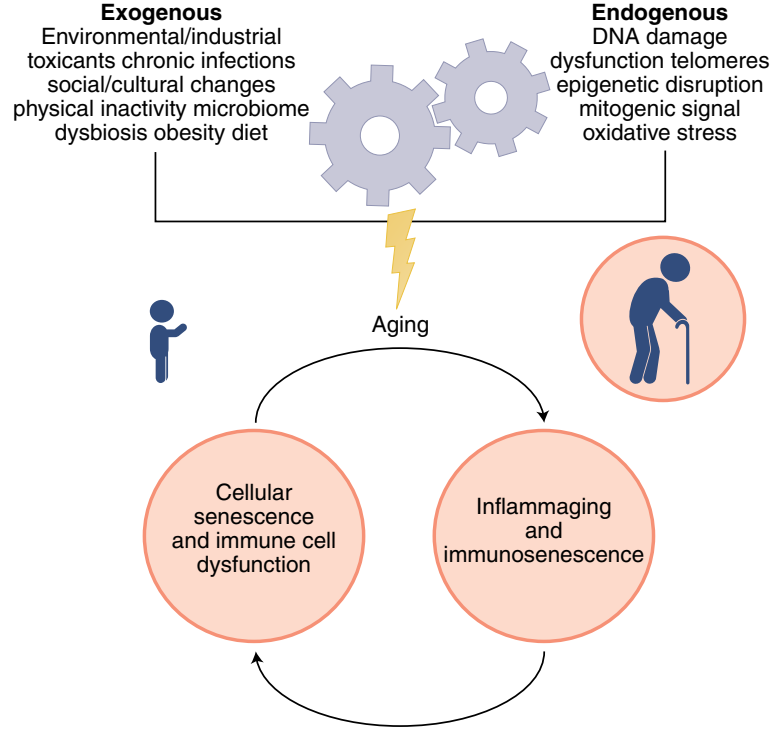

Fig. 1 | Factors that contribute to inflammaging and immunosenescence. Both exogenous and cell-endogenous factors contribute to chronic inflammation with age, which is primarily mediated by immune cells and senescent cells. Senescent cells persist and accumulate during aging, and they display an abnormal secretory phenotype, characterized by the production of inflammatory mediators, matrix metalloproteinases, fibronectin and ROS. These inflammatory mediators contribute to inflammaging, which, over time, affects immune cell function, promoting immunosenescence. The endogenous and exogenous factors listed here can also directly affect the inflammatory potential of the immune system, which further promotes a feed-forward loop of inflammaging and immunosenescence.

an impairment in antigen presentation and naive $\mathrm{T}$ cell priming, a propensity for myeloid lineage differentiation in the bone marrow, altered type I interferon (IFN) responses, diminished $\mathrm{CD}^{+} \mathrm{T}$ cell cytotoxic function, decreased phagocytic function for many innate immune cell types, a restricted naive $\mathrm{T}$ cell and $\mathrm{B}$ cell repertoire and impaired production of high-avidity antibodies ${ }^{29,30}$. These immunological changes weaken immune responses to most viruses, leaving older individuals particularly vulnerable to influenza, SARS-CoV-2 and other lethal coronaviruses, such as SARS-CoV-1 and Middle East respiratory syndrome (MERS)-CoV ${ }^{31}$. The effects of aging on the human immune system have been extensively reviewed elsewhere ${ }^{30,32-34}$; thus, we will focus on aspects of the aging immune system that specifically overlap with characteristics of the immune response during severe COVID-19 outcomes.

\section{COVID-19: an overview in pathogenesis}

SARS-CoV-2 is the causative agent of COVID-19, declared a global pandemic by the World Health Organization on March 11, 2020 (ref. ${ }^{35}$ ). SARS-CoV-2 has a single-stranded, positive-sense RNA genome ${ }^{36}$. Attachment and entry of SARS-CoV-2 to target cells is initiated when the virus engages its cognate receptor, angiotensin-converting enzyme 2 (ACE2), via the receptor-binding domain (RBD) of the viral spike protein, also called the $S$ protein ${ }^{37}$. Host transmembrane protease serine 2 (TMPRSS2) promotes $S$ protein priming and facilitates viral entry ${ }^{38}$. Primary cell types for SARS-CoV-2 entry into the body include nasal epithelium, alveolar type II pneumocytes, superficial conjunctival cells and many types of enterocytes in the gut ${ }^{39}$. Virus entry into such cells leads to viral replication, destruction of infected cells and triggering of an innate immune response. These processes occur early during the SARS-CoV-2 incubation period of approximately
$5 \mathrm{~d}$. SARS-CoV-2 is likely to be initially detected by host cells via Tolllike receptor (TLR)7 and TLR8, which sense single-stranded RNA, and potentially TLR3, which senses double-stranded RNA intermediates ${ }^{40}$. TLR signaling engages IFN-regulatory factors (IRFs) and MyD88$\mathrm{NF}-\kappa \mathrm{B}$ signaling pathways, leading to the production of type I IFNs and pro-inflammatory cytokines (interleukin (IL)-6, tumor necrosis factor (TNF)- $\alpha$ and IL- $1 \beta)^{40}$. Host cell damage resulting from SARSCoV-2 infection can also lead to release of endogenous damage-associated molecular patterns (DAMPs), such as ATP, DNA and oxidized phospholipids $^{41}$. DAMPs serve as danger signals for inflammasomes, multiprotein immune complexes that cleave and activate IL- $1 \beta$ and IL-18, and are also directly sensed by PRRs including TLRs to further propagate inflammation ${ }^{40,42}$. Pro-inflammatory cytokine and chemokine production recruits and activates innate immune cells, including neutrophils, natural killer cells, dendritic cells (DCs) and monocytes. Activated DCs and viral antigens eventually migrate to the draining lymph nodes, where they engage the adaptive immune system, T cells and $\mathrm{B}$ cells, with potential to clear the virus.

After the viral incubation period, in most cases, a young healthy individual will clear the virus with this coordinated immune response. However, patient symptoms can vary greatly, from asymptomatic to severe, with the latter being more common in older adults ${ }^{43,44}$. Patients with severe COVID-19 have higher levels of circulating cytokines and chemokines, contributing to enhanced risk for critical outcomes of COVID-19: pneumonia, cytokine storm, $\mathrm{ARDS}^{45}$, sepsis and coagulopathy ${ }^{46,47}$. ARDS is a severe and often fatal complication of COVID-19. It is clinically defined by the acute onset of respiratory failure, hypoxemia, bilateral lung infiltrates on chest imaging not fully explained by effusions, collapse and/or nodules and lack of cardiogenic-related edema $^{48}$. During COVID-19, ARDS manifests as a diffuse alveolar damage pattern seen in autopsies of patients with COVID-19 and is linked to a combination of lung immune cell infiltration, cytokine storm and tissue damage from secreted proteases, reactive oxygen species (ROS) and viral killing ${ }^{49,50}$. Cytokines that mediate manifestations of ARDS, such as IL- 6 and TNF- $\alpha$, can also facilitate vascular permeability, systemic shock and multiorgan failure ${ }^{41,51}$. Many patients with severe COVID-19 also develop bacterial pneumonia, a secondary bacterial infection of the lungs ${ }^{52}$. These bacterial infections bolster pro-inflammatory responses that perpetuate cytokine storm, systemic inflammation and ARDS phenotypes ${ }^{53}$.

Mouse studies of SARS-CoV-2 show a higher viral burden in older mice in the first few days after infection, suggesting that aging may also lead to a delay or dysfunction in the initial triggering and priming stages of the immune response ${ }^{54,55}$ that is propagated in part by suboptimal antigen-specific adaptive immunity ${ }^{56}$. The onset of ARDS in severe cases typically occurs around day 12 after symptom onset ${ }^{44,57}$, when viral shedding appears to be mostly resolved ${ }^{58}$, followed by death or recovery around 7-12 d later ${ }^{44,57}$. Therefore, severe cases of COVID-19 are not simply due to an inability to clear the viral infection but rather due to a sustained, dysregulated and highly destructive inflammatory response. A basic summary of clinical progression and associated immune responses during mild and severe COVID-19 is depicted in Fig. 2.

\section{Immune system dysregulation associated with aging and severe COVID-19: innate immunity}

Primary goals of the innate immune system in response to a viral infection are (1) to initiate a local inflammatory response to activate and recruit immune cells, (2) to directly eliminate virally infected cells and (3) to prime the adaptive immune response. As we age, the ability to achieve these three goals is either diminished or dysregulated. Here, we discuss components of the innate immune system, describe inflammatory processes implicated in both inflammaging and severe COVID-19 and provide a summary in Fig. 3. 


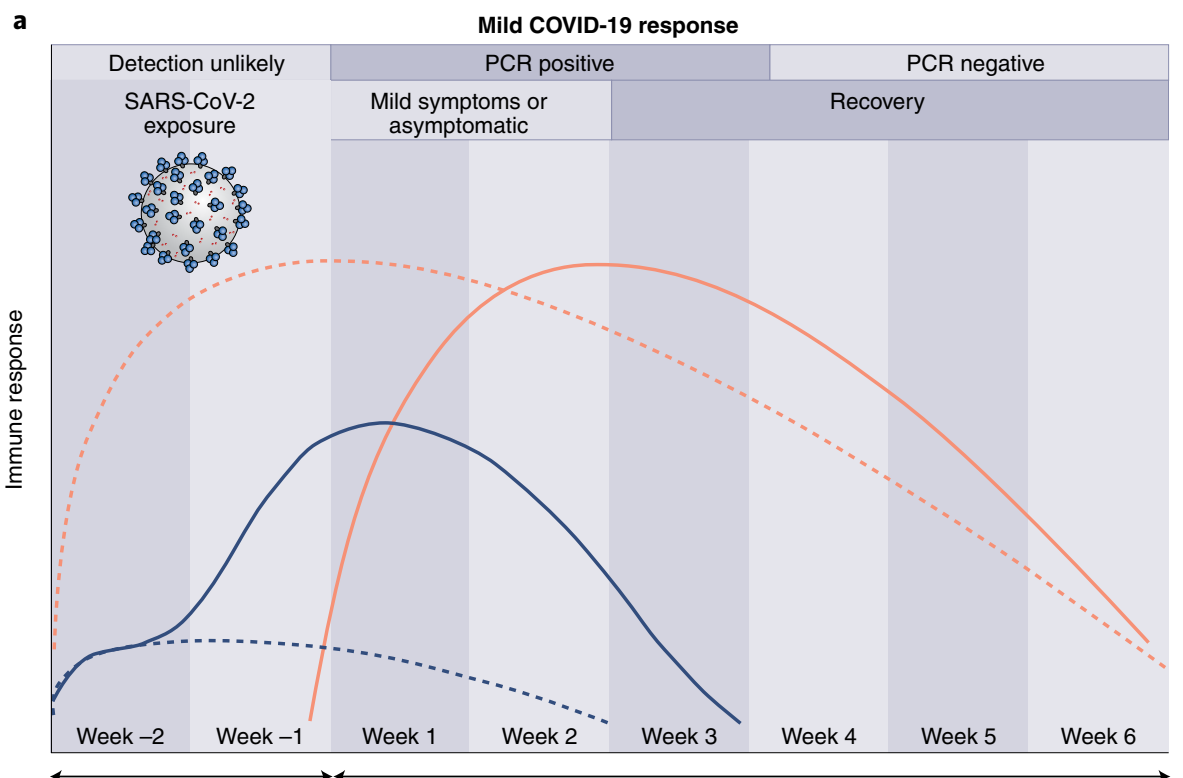

Before sympton onset

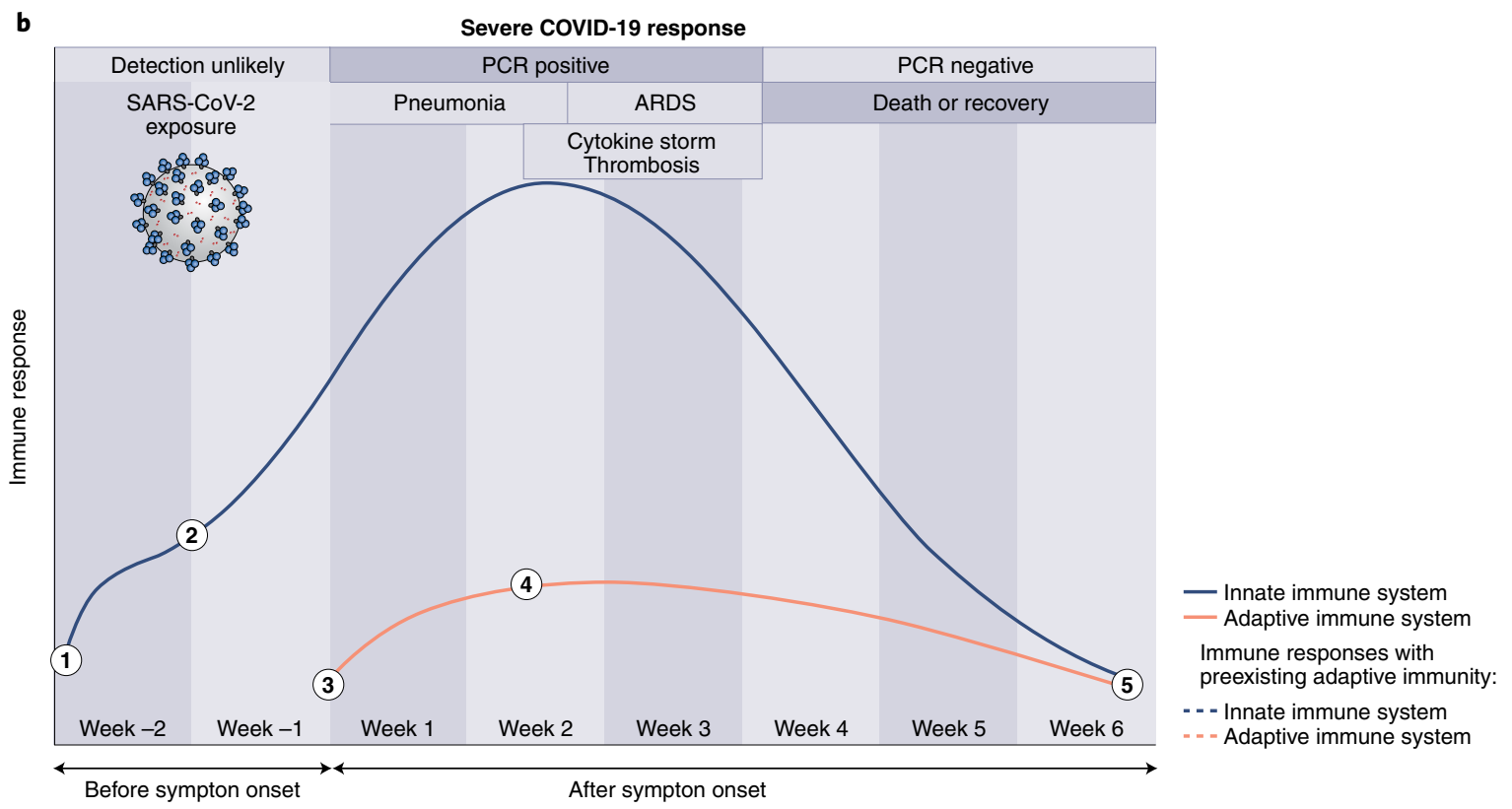

Fig. 2 | Immune response patterns and clinical disease courses for mild and severe COVID-19. For mild COVID-19 (a) and severe COVID-19 (b), graphs show the general disease course timeline and the magnitude of innate (blue) and adaptive (orange) immune responses over time. Dashed lines represent the time course for response in patients with pre-existing adaptive immunity. For severe COVID-19 (b), there are five main points of interest in the immune response that could be influenced by an aging immune system: (1) heightened basal inflammation (inflammaging) at the point of SARS-CoV-2 inoculation may predispose older adults to an already pro-inflammatory local environment; (2) innate immune cell dysfunction associated with aging may alter early immune responses; (3) delayed and/or diminished adaptive responses due to poor naive clonal diversity, weak or ineffective pre-existing immunity and poor T cell priming; (4) altered T cell effector function and antibody responses due to immunosenescence; and (5) the possibility of a reduced memory response and potential long-lasting effects on the immune system that may further promote immunosenescent phenotypes.

Anti-inflammatory effects of ACE2. The primary SARS-CoV-2 cellular receptor ACE2 can play a direct role in early inflammatory processes through the renin-angiotensin-aldosterone system signaling pathway, thereby aiding in one of the primary goals of the innate immune system: initiating a local inflammatory response to activate and recruit immune cells. ACE2 converts angiotensin II (an inflammatory mediator) to angiotensin 1-7 (an anti-inflammatory mediator). Angiotensin II signaling generates a pro-inflammatory state in vascular cells, leading to enhanced vascular permeability and local inflammation ${ }^{59}$. In mouse models of ARDS, the antiinflammatory effect of ACE2 was shown to be protective against severe acute lung injury ${ }^{60}$. Angiotensin 1-7 was also shown to decrease the production of pro-inflammatory cytokines, specifically IL-6, TNF- $\alpha$ and IL-8, through inhibition of the p38-mitogen-activated protein kinase (MAPK)-NF- $\kappa$ B signaling pathway while also upregulating expression of the anti-inflammatory cytokine IL-10 $\left(\right.$ ref. $\left.{ }^{61}\right)$. An increased presence of angiotensin II during the early response to SARS-CoV-2 has been postulated to play a role in 


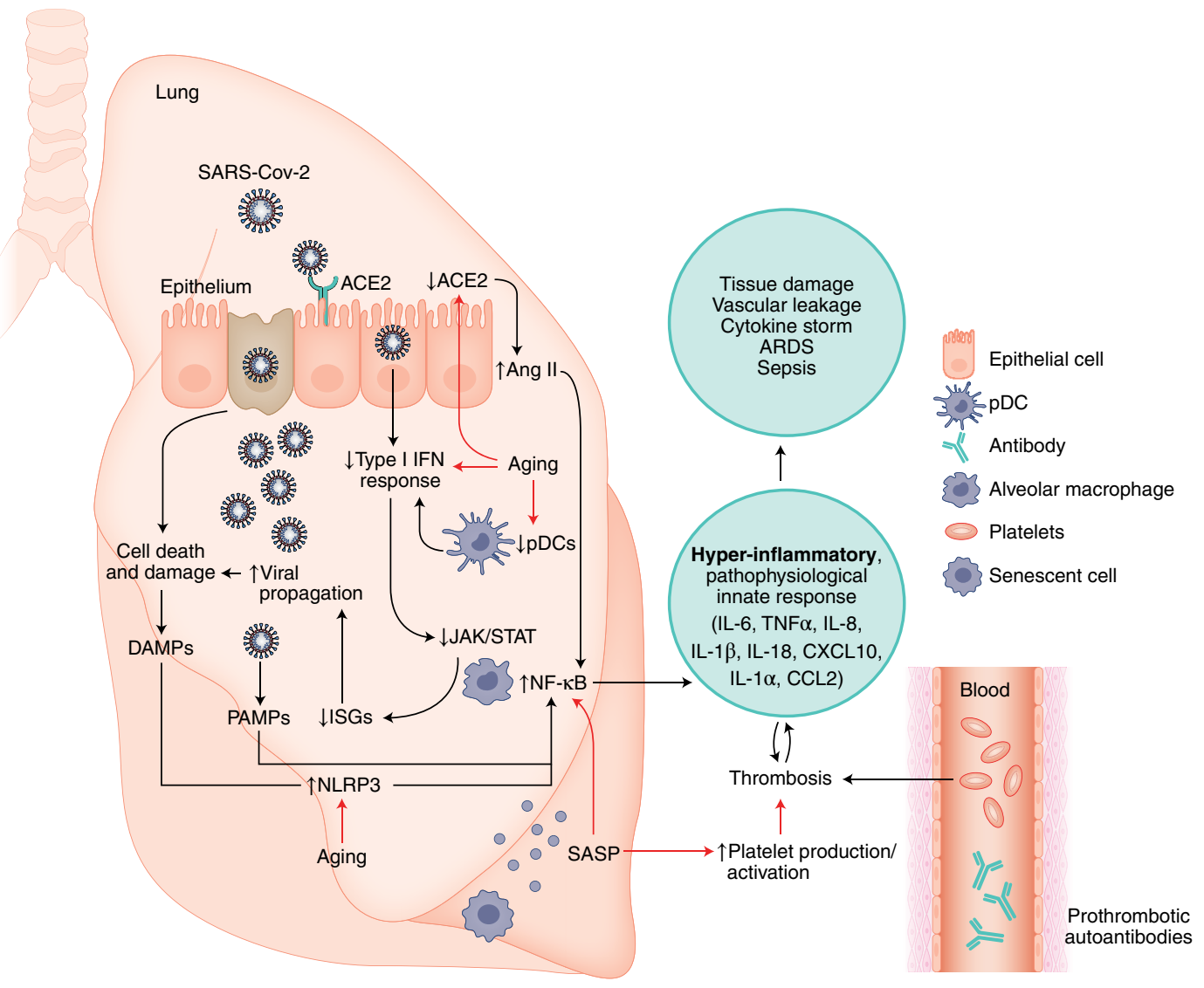

Fig. 3 | An aging innate immune system may predispose a patient to severe COVID-19. Consequences of the virus are indicated by black arrows, while consequences of aging are indicated by red arrows. SARS-CoV-2 first enters cells by binding to ACE2, and viral entry then leads to the shedding of ACE2. Aging may also be associated with reduced expression of ACE2, and low expression of ACE2 after infection potentially mediates a pro-inflammatory state through the production of angiotensin (Ang) II. Severe COVID-19 has also been correlated with reduced type I IFN responses, a defect that may be compounded by aberrant type I IFN signaling with age. This could be made worse by the notable reduction in the total number of pDCs found in patients with severe COVID-19. Aged pDCs also have a reduced ability to produce type I IFNs, and the pro-inflammatory nature of the SASP in older individuals desensitizes JAK-STAT signaling in innate immune cells. All of this would compound the loss of type I IFN signaling during severe COVID-19, ultimately leading to reduced expression of antiviral IFN-stimulated genes (ISGs) and permitting enhanced viral propagation. This viral replication leads to cell death and damage, causing the production of immunostimulatory DAMPs and PAMPs. Signaling in response to these signals generally activates the NF- $\mathrm{KB}$ transcription factor, which is also the main immune signaling pathway engaged by SASP mediators and the persistent basal stimulation of PRRs in older adults. NF- $\mathrm{kB}$ signaling promotes production of pro-inflammatory cytokines. DAMPs linked to both aging and viral infection can also trigger activation of inflammasomes such as NLRP3, potentiating cytokines such as IL-1 $\beta$ and IL-18, as well as triggering further cell death through pyroptosis. SASP mediators and the autoimmune-prone environment associated with aging may also predispose aged individuals to a pro-thrombotic environment, and thrombosis and inflammation continue to promote one another in a feed-forward loop, which further contributes to the hyperinflammatory, pathophysiological innate response that causes the devastating outcomes observed in severe COVID-19.

promoting cytokine storm phenotypes associated with severe COVID-19, probably because of excessive production of TNF- $\alpha$ and local macrophage activation ${ }^{62}$. Interestingly, there is a reduction in ACE2 expression in lungs of old rats when compared to those of their younger counterparts ${ }^{63}$, and one study showed a decrease in ACE2 mRNA in multiple tissues in older humans when compared to that in their younger counterparts ${ }^{64}$. ACE2 is also downregulated in humans with cardiovascular disease and diabetes, which also correlate with severe COVID-19 (ref. ${ }^{65}$ ). Decreased expression of ACE2 could mean less potential for SARS-CoV-2 invasion of host cells, but further reduction of ACE2 levels caused by viral entry may exacerbate a heightened pro-inflammatory response that helps to mediate cytokine storm, acute lung injury and ARDS. This hypothesis is supported by research showing elevated levels of angiotensin II in the plasma of patients with severe COVID-19 (ref. ${ }^{66}$ ). Moreover, a study of SARS-CoV-2 infection in old and juvenile rhesus macaques showed lower levels of ACE2 in old macaques than in young macaques after SARS-CoV-2 infection, supporting the hypothesis that ACE2 is dysregulated in older individuals, even across species ${ }^{67}$.

A propensity for pro-inflammatory cytokine production. An uncontrolled inflammatory response to SARS-CoV-2 can lead to harmful and even irreversible tissue damage, both locally and systemically. The majority of patients with severe COVID-19 demonstrate substantially elevated serum levels of pro-inflammatory cytokines and chemokines, including IL-6, IL-1 $\beta$, IL-2, IL-8, IL-17, granulocyte (G)-colony-stimulating factor (CSF), granulocyte-macrophage (GM)-CSF, C-X-C motif chemokine ligand (CXCL)10, C-C motif chemokine ligand (CCL)2, CCL3 and $\mathrm{TNF}^{49,54,68}$. In mouse studies of SARS-CoV-2 in which aging mice were directly investigated, similar cytokines, including IL-6, IL- $1 \alpha$, IL- $1 \beta$, TNF- $\alpha$ and the chemokine CCL2 were also linked to the aged host response to SARS-CoV-2 (refs. ${ }^{54,55}$ ). Many of these cytokines are also elevated basally in older individuals owing to inflammaging. For instance, elevated IL-6 levels have been an especially stable 
indicator of poor outcomes in patients with COVID-19, and studies have demonstrated that IL- 6 is also among the most reliable aging parameters ${ }^{69}$. The presence of IL-6 is a clinical hallmark of vascular activation of $\mathrm{NF}-\mathrm{KB}^{70}$, a major transcription factor that regulates many pro-inflammatory genes of innate immune cells. There is a close link between aging, NF- $\mathrm{B}$ signaling and inflammation ${ }^{71}$. Moreover, an anti-IL- 6 receptor antibody could attenuate COVID19 severity in a cohort of predominantly older patients (median age, 67 years), pointing to some cytokines as important drivers of exaggerated disease $\mathrm{e}^{72}$. IL- $1 \beta$ and IL-18 are also critical in inflammaging; these cytokines are products of the NLR-family pyrin domaincontaining (NLRP) 3 inflammasome and contribute to the pathology of aging-related diseases ${ }^{73}$. In aging patients, SARS-CoV-2 can potentiate NLRP3 inflammasome activation and IL-1 $\beta$ and IL-18 levels, pyroptosis and the release of DAMPs (including ATP), further heightening inflammation and COVID-19 pathogenesis ${ }^{74,75}$. ROS levels increase with age owing to mitochondrial dysfunction and inflammation and can also drive NLRP3 inflammasome activation, which is likely to further contribute to COVID-19 pathophysiology in older patients ${ }^{58,76,77}$. Interestingly, metformin may lower risk of severe COVID-19, as it reduces ATP and mitochondrial ROS levels, which can fuel NLRP3-mediated IL-1 $\beta$ production $^{78}$. Additionally, treatment with an IL-1 receptor antagonist has led to improvements in mortality in severe COVID-19 (ref. ${ }^{79}$ ), and the NLRP3 inflammasome is considered an emerging target for COVID-19 (ref. ${ }^{80}$ ).

Despite elevated NF- $\mathrm{KB}$ signaling observed at baseline during aging, many immune cells (including B cells, T cells and DCs) isolated from an inflammaging environment were shown to be hyporesponsive to acute ex vivo stimuli, although monocytes appear to stand out as being hyper-responsive ${ }^{81}$. Consistent with these observations, mass cytometry and single-cell RNA-seq analysis of monocytes from older patients with COVID-19 showed that they were enriched for the IFN- $\gamma$ response and the expression of TNF- $\alpha$, IL- $1 \beta$ and CXCL8 $\left(\right.$ ref. ${ }^{76}$ ). Aged monocytes were also enriched for broad pathways linked to TLR signaling, oxidative stress, MAPK and $\mathrm{NF}-\mathrm{\kappa B}$, as well as the senescent cell marker p21, leading the authors to speculate that the presence of inflammatory aged or senescent cells with a senescence-associated secretory phenotype (SASP) was one mechanism for exuberant inflammatory tone in these cells during COVID-19 (ref. ${ }^{76}$ ). Indeed, senescent cells persist and accumulate during aging ${ }^{82}$, and they display an abnormal secretory phenotype characterized by the presence of inflammatory cytokines that contributes to inflammaging. This SASP is characterized by the production of cytokines, chemokines, growth factors, matrix metalloproteinases, fibronectin and $\mathrm{ROS}^{83}$. In support of a role of cellular senescence in COVID-19 pathology, a recent study showed that in vitro exposure of senescent mouse and human cells to pathogenassociated molecular patterns (PAMPs) and the viral S protein leads to significantly increased SASP production and expression of the viral entry genes ACE2 and TMPRSS2, compared to non-senescent cells. Using a $\beta$-coronavirus mouse hepatitis virus that has some relation to SARS-CoV-2, the study demonstrated that targeting senescent cells in genetically modified mice and with senolytics (fisetin and dasatinib with quercetin) could reduce inflammation and improve survival ${ }^{84}$.

Neutrophils from older individuals exhibit some hyporesponsive attributes including: decreased bactericidal activity, decreased respiratory burst and decreased neutrophil extracellular trap formation $^{85}$. However, neutrophils from older adults also display aberrant migration and enhanced degranulation, suggesting that neutrophils of aged individuals are not able to fight off pathogens efficiently but still produce highly inflammatory and damaging molecules in a potentially non-localized fashion ${ }^{86}$. Moreover, elevated levels of IL-6, either as part of the host pathogen response or due to inflammaging, engender prolonged neutrophil survival by reducing apoptosis $^{87}$, and neutrophilia is in fact an indicator of poor clinical outcomes for COVID-19 (ref. ${ }^{88}$ ).

Thus, aged individuals may have dysregulated innate immune cell function and inflammatory responses to SARS-CoV-2. Consistently, studies have shown that the sustained presence of increased neutrophils and monocytes in the blood is associated with severe COVID-19 disease ${ }^{89,90}$. As mentioned, these changes occur in part due to age-related high basal TLR activation, changes in oxidative stress pathways, inflammasome activation, increased senescent cell burden and the SASP, as well as other pathways such as reduced autophagy and DNA damage with age ${ }^{91}$. Thus, SARS-CoV-2 infection combined with inflammaging leads to an exaggerated innate immune response and worsened outcomes of COVID-19. However, more studies are needed to tease apart the complex interconnections between inflammaging and susceptibility to severe COVID-19.

The pro-thrombotic nature of senescent cells and severe COVID-19. While the connection between the pro-inflammatory profile of senescent cells and the exuberant pro-inflammatory environment of severe COVID-19 is important to note, there is another intriguing connection between the two: thrombosis. Senescent cells have a paracrine pro-coagulation effect ${ }^{92}$. Induction of cellular senescence via doxorubicin treatment in a senescence reporter mouse, p16-3MR, was associated with significantly shorter tail bleed times, greater platelet count, more highly activated platelets and higher levels of thrombopoietin in the serum ${ }^{92}$. Elimination of senescent cells in vivo reversed these pro-thrombotic phenotypes. Stable isotope labeling by amino acids in cell culture analysis of human senescent cells and ex vivo experiments also showed that human platelets sensitized by senescent cell supernatants were more highly activated ${ }^{92}$.

Pro-thrombotic coagulopathy, high levels of $\mathrm{D}$-dimer, venous thromboembolism, arterial thromboses and fibrin-based occlusion of small blood vessels have all been associated with cases of severe COVID-19 (refs. ${ }^{7,44,49,93-99}$ ). Mechanistically, pro-inflammatory cytokines lead to the expression of tissue factor (CD142), the initiator of blood coagulation, on platelets, monocytes, macrophages and endothelial cells. Complement activation, neutrophil extracellular traps and lung hypoxia further propagate pro-thrombotic conditions, which work in a feed-forward loop with ongoing inflammation, connecting thrombosis and the innate immune system ${ }^{100}$. A study also described the production of viral-induced pro-thrombotic autoantibodies against phospholipids and phospholipidbinding proteins during cases of severe SARS-CoV-2 infection ${ }^{101}$. Thus, the combination of a heightened inflammatory response to SARS-CoV-2 with the already thrombotic-prone microenvironment associated with aging may further potentiate the coagulopathy associated with worsened outcomes of COVID-19.

Loss of type I IFN responses. Reduced type I IFN levels have been observed in the serum of patients with life-threatening COVID-19 (ref. ${ }^{102}$ ). In a rhesus macaque model of SARS-CoV-2 infection, old macaques also had reduced type I IFN and Notch signaling pathways in their lungs when compared to their juvenile counterparts ${ }^{67}$. Reduced type I IFN responses in humans are in part linked to a reduction in numbers of plasmacytoid DCs (pDCs), strong producers of IFN- $\alpha$, which has also been reported in cases of severe COVID-19 (ref. ${ }^{103}$ ). Ten per cent of all patients with life-threatening COVID-19, however, also harbor autoantibodies against type I IFNs ${ }^{104}$. Moreover, patients with severe symptoms can also carry loss-of-function variants in multiple genes linked to TLR3 and IRF7 pathways, involved in both the induction and amplification of type I IFNs ${ }^{105}$. If type I IFNs are present early and properly localized to sites of infection, they can effectively limit viral propagation, as type I IFNs are responsible for optimal activation of macrophages, antigen presentation by DCs and enhanced antiviral effector $\mathrm{T}$ cell responses ${ }^{106}$. Likewise, IFN-induced transmembrane family 
(IFITM) proteins may inhibit SARS-CoV-2 entry, as demonstrated for previous coronaviruses ${ }^{107,108}$.

Studies have revealed the importance of type I IFN responses in regulating monocytes and neutrophils early after SARS-CoV-2 infection. Peripheral blood from patients with mild COVID-19 contained more classical monocytes $\left(\mathrm{CD} 14^{+} \mathrm{CD} 16^{-}\right)$exhibiting an early and transient type I IFN signature ${ }^{109,110}$. Conversely, monocytes and neutrophils from patients with severe COVID-19 expressed more genes involved with NF- $\kappa \mathrm{B}$ signaling and ROS or nitric oxide synthase production throughout the course of the disease ${ }^{109}$. Nonclassical $\left(\mathrm{CD} 14^{+} \mathrm{CD} 16^{++}\right)$and intermediate $\left(\mathrm{CD} 14^{++} \mathrm{CD} 16^{+}\right)$subsets of monocytes are pro-inflammatory and known to expand during viral infections ${ }^{111}$. Thus, their expansion in response to SARS-CoV-2 infection is expected ${ }^{112,113}$; however, a dysregulated exuberant response from these cells would also contribute to the highly inflammatory environment observed in severe COVID-19 pathophysiology. Intriguingly, non-classical monocytes were also shown to produce the type I IFN IFN- $\alpha$ in response to TLR3 (ref. ${ }^{114}$ ). Thus, it is important to consider the kinetics of the dysregulation of non-classical monocytes in severe outcomes, as an early loss of this subset of monocytes has been described by some ${ }^{109,110}$ and may further contribute to the reduced type I IFN response observed in patients with severe COVID-19.

Aging leads to a delay in type I IFN responses, linked to changes in viral sensing, which was also observed for SARS-CoV-1 (ref. ${ }^{31}$ ). While the mechanisms of this are not entirely known, aging compromises both primary and secondary retinoic acid-inducible gene (RIG)-I signaling pathways that control expression of many type I IFN genes ${ }^{115}$. This is associated with reduced production of type I IFNs in those $>65$ years of age, impairing their antiviral responses, similar to that observed with respiratory influenza $\mathrm{A}$ virus $^{115}$. During SARS-CoV-1 infection, levels of type I IFN signaling proteins downstream of RIG-I are further reduced due to infection-induced mitochondrial dysfunction linking to the viral sensor MAVS $^{116}$. Given basal mitochondrial dysfunction, as well as a reduction in TNF receptor-associated factor (TRAF) adaptor protein and phosphorylated IRF3 levels linked to RIG-I signaling in older adults ${ }^{15,117}$, they are particularly vulnerable to such RIG-I insufficiency. Interestingly, RIG-I can restrain SARS-CoV-2 replication in human lung cells, although the mechanism does not require its type I IFN signaling ability ${ }^{118}$. Older individuals also display reduced total numbers of pDCs at baseline, and their pDCs often show reduced TLR7 expression ${ }^{117}$ and have diminished capacity to produce type I IFNs ${ }^{119-121}$. While there is a strong overlap of reduced type I IFN responses between older adults and patients with severe COVID-19, additional mechanistic connections between the two require further investigation.
Decline in antigen presentation and $T$ cell priming. Innate immunity plays a critical role in the initiation of adaptive immune responses by ensuring proper activation of Tlymphocytes. To achieve effective T cell priming, innate immune cells must accomplish two tasks: (1) present antigen via major histocompatibility complex (MHC) molecules alongside co-stimulatory receptors via cell-cell interactions between the $\mathrm{T}$ cell and the antigen-presenting cell (APC) and (2) produce the proper cytokines to skew $\mathrm{CD} 4^{+} \mathrm{T}$ cell differentiation toward the appropriate effector response specific to the invading pathogen. Defects in the ability of APCs to accomplish either of these tasks can have detrimental effects on the adaptive immune response and alter disease outcome.

APCs, such as DCs and monocytes, taken from the blood of patients with acute COVID-19 display impaired antigen-presenting abilities. When stimulated ex vivo, DCs taken from the blood of patients with COVID-19 had minimal expression of CD80, CD86, $\mathrm{C}-\mathrm{C}$ motif chemokine receptor (CCR)7 and human leukocyte antigen (HLA)-DR ${ }^{103}$. HLA-DR expression on monocytes from patients with severe COVID-19 is also reduced relative to that from patients with mild symptoms $s^{109,110,112,122}$. Aging negatively affects antigen presentation as well. Monocyte populations shift with aging, and there is an accumulation of non-classical monocytes that significantly downregulate HLA-DR ${ }^{123}$. Other work suggests reduced levels of MHC class II, CD40 and CD86 on aged DC subsets after activation with TLR agonists; although there are conflicting reports, as previously reviewed ${ }^{124}$. During SARS-CoV-1 infection, lung DCs from older mice displayed an impaired ability to migrate to the draining lymph node, which negatively affected subsequent $\mathrm{T}$ cell priming ${ }^{125}$. This migration defect was caused by increased levels of prostaglandin D2 in elderly mouse lungs, which directly reduced the surface expression of CCR7 on DCs ${ }^{125}$.

Aging predisposes an individual to impaired antigen presentation and $\mathrm{T}$ cell priming. Thus, a virus that further reduces the immune system's capacity for these essential tasks could render older adults especially susceptible to worse disease outcomes due to improper adaptive immune responses.

\section{Immune system dysregulation associated with aging and severe COVID-19: adaptive immunity}

The role of adaptive immunity in controlling SARS-CoV-2 infection has been a major scientific focus, as generating robust immunological memory is the primary goal of current vaccines. Adaptive immunity is carried out by three broad populations of lymphocytes: $\mathrm{B}$ cells, $\mathrm{CD} 4^{+} \mathrm{T}$ cells and $\mathrm{CD} 8^{+} \mathrm{T}$ cells. $\mathrm{B}$ cells are responsible for producing antiviral antibodies. $\mathrm{CD}^{+} \mathrm{T}$ cells act as 'helper' cells, producing cytokines to bolster the antiviral immune response and aid $\mathrm{B}$ cells in the production of long-lasting, neutralizing

Fig. 4 | Aging adaptive immune system responses during severe COVID-19. a-d, It has been hypothesized that a delayed and/or weak adaptive immune response helps to drive the dysregulated and continued recruitment of neutrophils and monocytes. These cells facilitate a nonspecific and highly destructive hyperinflammatory response that contributes to the outcomes observed in patients with severe COVID-19. Failures of the adaptive system would begin at sites of infection (a), where DCs are initially activated by PAMPs and DAMPs in response to SARS-CoV-2. Patients with severe COVID-19 have DCs with a reduced maturation profile. $\mathbf{b}$, Aging also negatively affects DC maturation, furthering the potential for improper priming of naive $T$ cells in lymph nodes in older adults. There appears to be a loss of GC reactions with atrophy of lymphoid follicles, associated with atrophy of secondary lymphoid organs (SLOs) in fatal cases of COVID-19. These factors, combined with decreased CD4OL expression on aged CD4+ T cells and reduced AID expression in aged B cells, could culminate in greatly impaired high-avidity, long-lasting antibody production that is dependent upon the success of $\mathrm{GC}$ reactions. Reductions in levels of $\mathrm{GC}$ reactions are also linked to extrafollicular and age-associated $B$ cell (ABC) responses, which may potentiate 'lupus-like autoimmune inflammation'. As we age, the naive TCR repertoire also decreases, making DC accessibility to SARS-CoV-2-specific naive T cells potentially even more challenging and delaying priming responses. c,d, Lymphopenia is also strongly associated with severe COVID-19 outcomes. The aged microenvironment may contribute to this phenotype, as the SASP mediators IL- 6 and TNF- $\alpha$ can cause enhanced T cell apoptosis. Furthermore, the presence of virtual memory CD8 ${ }^{+} \mathrm{T}$ cells at sites of SARS-CoV-2 infection may contribute to a hyperinflammatory response through the action of bystander cytotoxicity, which occurs in a non-antigen-specific manner and can be highly destructive if left unregulated. AID, activation-induced cytidine deaminase; FDC, follicular dendritic cells; $S H M$, somatic hypermutation; $T_{\text {eff }}$ cell, effector $T$ cell. Immune responses to the virus are indicated by black arrows, and consequences of aging are shown by red arrows. 
antibodies. $\mathrm{CD}^{+} \mathrm{T}$ cells directly kill virally infected cells while also producing antiviral cytokines. Collectively, these three arms of the adaptive immune response have proven critical in controlling viral infections, including that with SARS-CoV-2. With age, adaptive immunity wanes, potentially allowing SARS-CoV-2 to evade or subvert this arm of the immune system. Here, we review adaptive immunological responses to SARS-CoV-2 and comment on how features of the aging immune system may contribute to a defective adaptive response during severe COVID-19. A summary is depicted in Fig. 4.

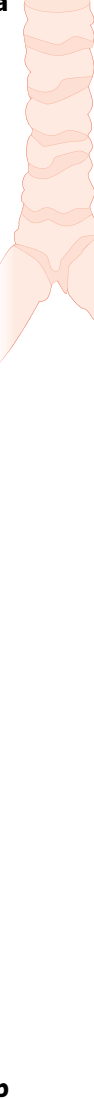

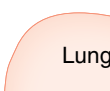

Lung
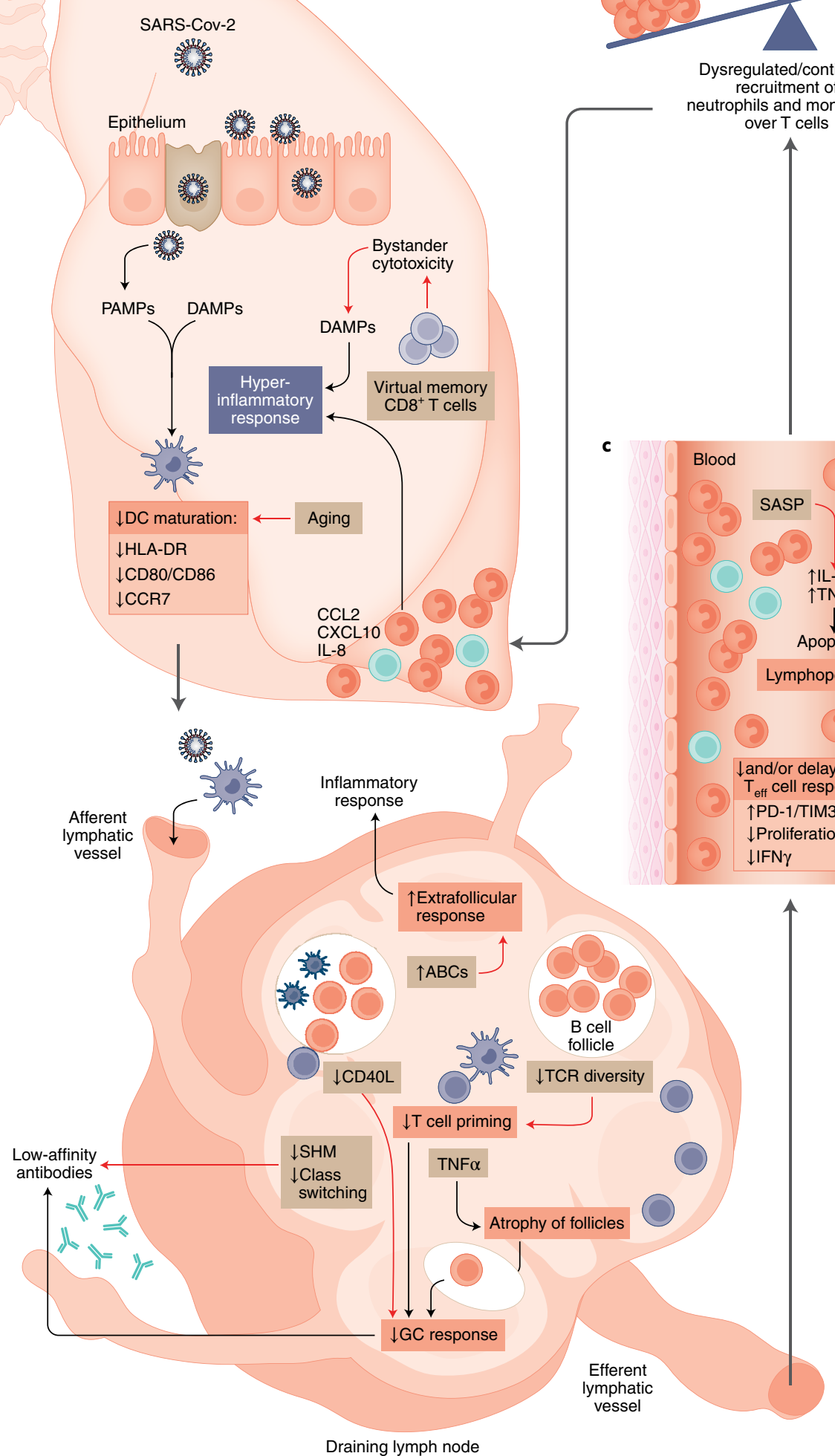

d

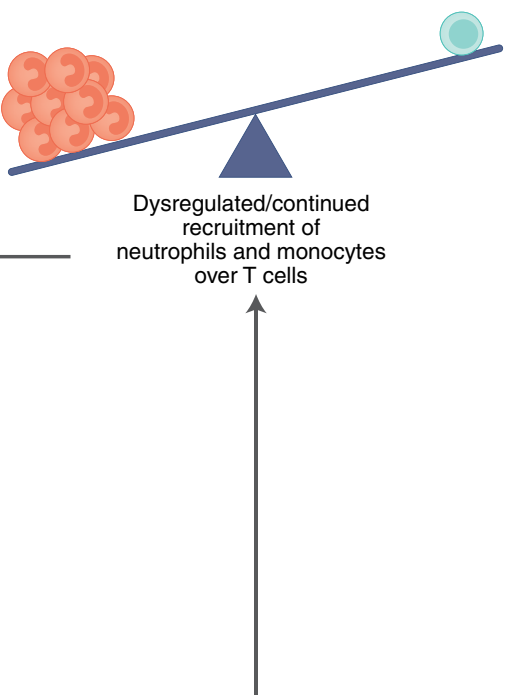

c

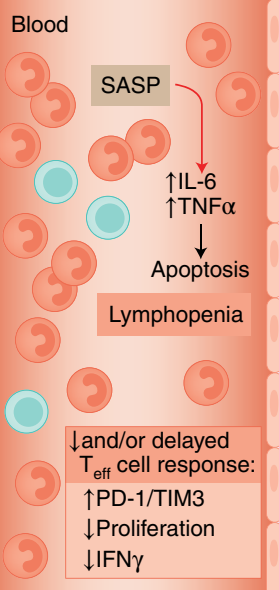

Epithelial cell

SARS-CoV-2

और $D C$

और

- Antibody

B cell

Naive T cell

$\mathrm{CD}^{+}$memory cells

$\mathrm{T}$ effector cell

Neutrophils and monocytes 
T lymphocytes. Adaptive responses to SARS-CoV-2 begin around 1 week after symptoms (Fig. 2), with SARS-CoV-2-specific CD $4^{+}$ and $\mathrm{CD}^{+} \mathrm{T}$ cells being observed as early as $4 \mathrm{~d}$ after symptom onset ${ }^{126}$. SARS-CoV-2 infection elicits a CD4 $4^{+}$type 1 helper $\mathrm{T}\left(\mathrm{T}_{\mathrm{H}} 1\right)$ cell response, with IFN- $\gamma$ and IL-2 being the primary cytokines produced by these cells, as well as some TNF- $\alpha^{112,126-129}$. Indeed, many chemokines that are elevated in the blood of patients with COVID-19, such as CXCL9 and CXCL10, are involved in recruiting and/or differentiating naive $\mathrm{T}$ cells into $\mathrm{T}_{\mathrm{H}} 1$ cells. Both $\mathrm{CD}^{+}$and $\mathrm{CD}^{+}$ $\mathrm{T}$ cells confer protection against previous coronaviruses ${ }^{130}$. Current evidence suggests that an early, robust and diverse $\mathrm{T}$ cell response directed against SARS-CoV-2 correlates with milder COVID-19 outcomes $^{126,131,132}$; however, excessive cytokine release from $\mathrm{T}$ cells could also contribute to aberrant activation of monocytes during cytokine storm. As aging impacts many aspects of T cell biology, it is important to understand how these changes may influence worsening outcomes of SARS-CoV-2 infection in the older population.

Reduced naive lymphocyte clonal diversity. Maintaining a diverse TCR repertoire is critical for optimal functioning of the immune system, but naive $\mathrm{CD}^{+}$and $\mathrm{CD} 8^{+} \mathrm{TCR}$ diversity diminishes as we age $^{133}$. There are many factors that may contribute to the restricted TCR repertoire in older adults, but the exact mechanisms underpinning this phenomenon are still debated. Thymic involution, a process by which the thymus gradually atrophies as we age, prevents development of new T cells in older individuals, thus restricting the TCR repertoire. Evidence suggests that newly generated naive T cells that do manage to enter the aged $\mathrm{T}$ cell repertoire display reduced $\mathrm{T}$ effector cell qualities upon activation and generate less effective memory cells ${ }^{134}$. Nascent $\mathrm{T}$ cell generation, however, may only be a part of the story, especially with regard to $\mathrm{CD}^{+} \mathrm{T}$ cells. In humans, tightly regulated homeostatic proliferation and active maintenance of $\mathrm{T}$ cell quiescence are imperative for a stable peripheral naive $\mathrm{T}$ cell compartment, and these two factors may play a prominent role in sustaining naive $\mathrm{T}$ cell diversity as we age $\mathrm{e}^{135}$. Additionally, chronic antigen stimulation can lead to an expanded, oligoclonal $\mathrm{T}$ cell memory pool that further exacerbates the diminished presence of polyclonal naive $\mathrm{T}$ cells.

Severe COVID-19 has been associated with lower TCR diversity against SARS-CoV-2 epitopes ${ }^{131,132}$. Specifically, when compared to those with mild COVID-19, patients with severe disease generated a weaker $\mathrm{T}$ cell response to the $\mathrm{N}$-terminal portion of the SARS$\mathrm{CoV}-2 \mathrm{~S}$ protein, a region that includes the critical $\mathrm{RBD}^{128}$. Low frequencies of naive $\mathrm{T}$ cells have also been correlated with severe COVID-19 outcomes ${ }^{126}$. These findings could suggest a connection between the diminished naive TCR repertoire of older individuals before SARS-CoV-2 infection and worsened disease outcome.

Lymphopenia. COVID-19 severity is associated with lymphopenia, and many studies have shown a correlation between poor disease outcome and reduced total numbers of peripheral $\mathrm{T}$ cells in the blood $^{136}$. It was even suggested that a patient's blood lymphocyte percentage can be used clinically as an independent prognostic measure to identify moderate, severe and critically ill disease trajectories to inform early therapeutic decisions ${ }^{137}$. Lymphopenia observed in severe COVID-19 may prove even more detrimental for aged individuals, as immunosenescence leads to poorer $\mathrm{T}$ cell responses that could be further exacerbated by a decline in $\mathrm{T}$ cell numbers.

Initially, it was speculated that COVID-19-associated lymphopenia may be a direct result of increased $\mathrm{T}$ cell migration to sites of infection. However, while lymphocytes do accumulate in the lungs of deceased patients with COVID-19, this migration probably does not completely account for the magnitude of observed lymphopenia. One group analyzing bronchoalveolar lavage fluid by single-cell RNA-seq found that the total number of $\mathrm{CD}^{+} \mathrm{T}$ cells with a tissueresident phenotype was actually greater in patients with moderate disease than that in those with severe disease ${ }^{138}$. The same study also concluded that lung macrophages present in bronchoalveolar lavage fluid of patients with severe COVID-19 expressed chemokines more likely to recruit inflammatory monocytes and neutrophils, while lung macrophages from patients with moderate COVID-19 expressed higher levels of $\mathrm{T}$ cell-recruiting chemokines, supporting the hypothesis that $\mathrm{T}$ cell migration to the lungs does not account for lymphopenia observed in the blood in severe disease ${ }^{138}$. Other hypotheses to explain the depletion of T cells during SARS-CoV-2 infection include direct infection of $\mathrm{T}$ cells with the virus and/or increased prevalence of activation-induced cell death in response to either cognate antigen stimulation or as an effect of the cytokine milieu $^{41}$. Lymphopenia does correlate with serum levels of IL-6, IL-10 or TNF- $\alpha^{139,140}$, and the IL-6 receptor antagonist tocilizumab increased the number of circulating lymphocytes in patients with COVID-19 (ref. ${ }^{122}$ ).

One cohort of 522 patients with COVID-19 revealed a strong association between lymphopenia and age in addition to the association between lymphopenia and disease severity, such that patients older than 60 years had the lowest total number of $\mathrm{T}$ cells in their blood $^{139}$. In the case of influenza virus, aged $\mathrm{CD} 8^{+} \mathrm{T}$ cells showed reduced expansion or proliferative capacity, and this decrease in virus-specific $\mathrm{CD}^{+} \mathrm{T}$ cells negatively affected viral clearance in older patients ${ }^{134}$. Studies aimed at further delineating a similar association between lymphopenia, aging, $\mathrm{T}$ cell expansive capacity and SARS-CoV-2 infection may be of great interest for future investigation.

Decline in effector T cell function and enhanced cellular exhaustion. $\mathrm{T}$ cell effector functions tend to decrease with age, while $\mathrm{T}$ cell exhaustion increases. Analysis of blood from patients with severe versus mild COVID-19 during the acute phase of illness has revealed contradictory reports about whether $\mathrm{T}$ cells are functioning properly following SARS-CoV-2 infection. Some studies have found reduced effector functions for $\mathrm{CD} 4^{+} \mathrm{T}$ cells, that is, IFN- $\gamma$, IL- 2 and/or TNF- $\alpha$ production, in patients with severe disease ${ }^{141}$, while others have shown no differences ${ }^{142}$. For $\mathrm{CD}^{+} \mathrm{T}$ cells, there are reports of diminished $\mathrm{CD} 8^{+}$cytotoxicity and cytokine production in severe cases ${ }^{126,132}$, while others report the opposite ${ }^{141}$, and still others find no differences ${ }^{142}$. The differences across many studies may be due in part to the timing of cytokine sampling. Given that aging reflects a state of impaired adaptive immunological function, we favor a model in which aging predisposes an individual to exhausted or compromised effector T cells with reduced cytokine production and/or cytotoxicity, compromising viral clearance during acute stages of the disease. Consistent with this hypothesis, one study identified the presence of IFN- $\gamma$-producing $\mathrm{CD}^{+} \mathrm{T}$ cells during acute stages of disease as the strongest predictor of mild COVID-19 outcomes ${ }^{126}$. Moreover, CD4 ${ }^{+}$and $\mathrm{CD}^{+} \mathrm{T}$ cells also display greater expression of exhaustion markers (programmed cell death protein (PD)-1, T cell immunoglobulin- and mucin domaincontaining protein (TIM)3, etc.) in severe COVID-19 cases than those in mild ones ${ }^{139}$; however, these markers may simply represent activation rather than functional exhaustion, as one paper recently suggested $^{143}$.

Pre-existing immunity to SARS-CoV-2. SARS-CoV-2-reactive CD4 ${ }^{+}$ $\mathrm{T}$ cells are observed in up to $40-60 \%$ of unexposed individuals ${ }^{127}$. One study used cell-sorting experiments to show that SARS-CoV2 -reactive $\mathrm{T}$ cells identified from SARS-CoV-2-unexposed individuals were primarily from the memory $\mathrm{T}$ cell compartment and they were also reactive to other human coronaviruses ${ }^{144}$. Therefore, many individuals have existing memory $\mathrm{T}$ cells that were generated from endemic human coronavirus infections and can cross-react with SARS-CoV-2 epitopes, known as SARS-CoV-2-cross-reactive memory $\mathrm{T}$ cells ${ }^{128}$. Another study concluded that severe COVID-19 
may even be associated with a lack of these pre-existing SARS-CoV2 -cross-reactive memory T cells in the TCR repertoire ${ }^{131}$.

Given the relationship between COVID-19 severity and age, these findings require further investigation. For instance, it will be important to know whether older individuals have been exposed to more human coronavirus infections and whether this exposure translates into robust memory $\mathrm{T}$ cell responses. Indeed, the lack of TCR diversity with age may prevent broad memory T cell development from prior exposures to 'common cold' endemic human coronaviruses. Memory T cells generated by the elderly may also not be maintained as well in the peripheral repertoire, perhaps due to niche competition from the effects of memory inflation ${ }^{145}$. COVID-19 severity has been linked to the incidence of cytomegalovirus (CMV), a primary pathogen associated with memory inflation, but more work is needed to establish how CMV and other latent viruses in older adults affect new memory $\mathrm{T}$ cell formation, function and longevity ${ }^{146}$.

As we age, $\mathrm{CD}^{+} \mathrm{T}$ cells can also lose their naive quiescent state and differentiate in response to IL-15 signaling without the presence of their cognate antigen. These 'virtual memory' $\mathrm{CD}^{+} \mathrm{T}$ cells have been most widely studied in mice ${ }^{147}$, but a human equivalent population has been identified $\left(\mathrm{CD} 45 \mathrm{RA}^{+} \mathrm{KIR}^{+} \mathrm{NKG} 2 \mathrm{~A}^{+}\right.$Eomes $\left.^{+}\right)$and similarly positively correlates with age $\mathrm{e}^{147}$. Virtual memory $\mathrm{CD} 8^{+} \mathrm{T}$ cells are innate-like in that they can become activated by cytokines (IL-15, IL-18 and type I IFNs) and mediate cytotoxic effects without the need for their cognate antigen during viral infections. While this could provide a benefit in clearance of the virus, it could also prove highly damaging to the host if left unregulated ${ }^{148}$. Whether or not these cells play a role in COVID-19 outcomes has yet to be studied.

B lymphocytes. In response to SARS-CoV-2 infection, B cells produce detectable levels of immunoglobulin (Ig)M, IgG and IgA antibodies specific to SARS-CoV-2 at around 1 week after symptom onset, and, by 2 weeks, the majority of patients seroconvert for IgG and IgM. This response occurs concurrently with the detection of circulating SARS-CoV-2-specific T follicular helper cells, suggesting a role for $\mathrm{T}$ cell-dependent antibody production ${ }^{126}$. Neutralizing antibodies specific to the RBD of SARS-CoV-2 have been discovered in both mice and humans ${ }^{149,150}$, and passive transfer of these antibodies reduces disease severity and offers protection in mouse models of SARS-CoV-2. Convalescent patient serum samples have also yielded promising results in the clinic ${ }^{151}$. However, the natural role that antibodies play during the course of COVID-19 progression remains contested.

Many studies have reported a correlation between higher IgG antibody titers and severe disease, but others have found either the opposite or no correlation between antibody production and disease severity ${ }^{126}$. These seemingly contradictory results might possibly be explained by the dual role played by antibodies during viral infections: although neutralizing antibodies are generally beneficial to viral clearance, the early production or prior existence of already circulating, non-neutralizing antibodies can lead to antibody-mediated enhancement of viral entry and induce a severe inflammatory response. This is called antibody-dependent enhancement of disease; however, there is currently no evidence supporting a role for antibody-dependent enhancement during SARS-CoV-2 infection $^{152}$. Interestingly, the presence of IgG antibodies with an afucosylated IgG Fc tail during acute SARS-CoV-2 infection have also been associated with severe COVID-19 outcomes, perhaps owing to their stronger pro-inflammatory activity via Fcy RIIIa ${ }^{153}$.

The quality of the humoral immune response declines with age, as aged $\mathrm{B}$ cells display a diminished potential to undergo somatic hypermutation ${ }^{154}$, which could prevent aged individuals from generating robust neutralizing antibody titers to aid the clearance of natural infection and generate effective immunity against reinfections. Consistently, levels of viral S protein-specific IgG during early time points of acute SARS-CoV-2 infection in rhesus macaques are reduced in older macaques compared to those in young ones, consistent with age crippling the formation of class-switched antibody titers needed to clear the viral infection ${ }^{155}$.

Another change in B cells that occurs in animal models and in humans with aging is the accumulation of age-associated B cells with unique properties ${ }^{156}$. In mice, these cells are promoted by TLR7 responses ${ }^{156}$, which are relevant, being a major PRR for SARSCoV-2. In humans, these cells are found in the late memory fraction ( $\mathrm{IgD}^{-} \mathrm{CD} 27^{-}$, also called double-negative B cells), secrete inflammatory mediators such as TNF- $\alpha$, IL- 6 and IL- 8 and have been implicated in autoimmune disease, chronic viral infections and, more recently, in COVID-19 (refs. ${ }^{156-158}$ ). During COVID-19, critically ill patients show expansion of such cells, which also express CD11c and T-bet in a skewed extrafollicular B cell response, with signatures of lupus-like autoimmune disease ${ }^{158}$.

Consistent with a dominant extrafollicular B cell response, studies have consistently reported defective germinal center (GC) responses in secondary lymphoid organs of patients who have succumbed to SARS-CoV-2 infection ${ }^{129,159}$. One of these studies also noted diminished GC formation that occurred concurrently with high levels of TNF- $\alpha$ in lymph node follicles, and TNF- $\alpha$ was previously demonstrated to inhibit follicular helper T cell differentiation and subsequent GC formation ${ }^{160}$. TNF- $\alpha$ production also increases as we age and could contribute to this observed phenotype ${ }^{161-163}$. Other hallmarks of aging also affect GC formation. Expression of CD40 ligand (CD40L), a co-stimulatory molecule critical for successful $\mathrm{T}$ cell and $\mathrm{B}$ cell interactions, decreases on aged $\mathrm{CD} 4^{+} \mathrm{T}$ cells $^{134}$. Additionally, lymphopenia observed in older adults as well as in patients with severe COVID-19 could imply a reduction in the availability of $\mathrm{CD}^{+} \mathrm{T}$ cells to engage with B cells. This dilemma would be heightened by the lack of TCR and BCR clonal diversity in aged individuals, as the probability of cognate $\mathrm{T}$ cell-B cell interactions would be greatly reduced.

Another consequence of aging is the loss of tolerance and the emergence of autoantibodies. Recent work on SARS-CoV-2 has linked life-threatening COVID-19 to the presence of autoantibodies specific to type I IFNs, especially in men ${ }^{104}$. These autoantibodies were found across multiple ages, although there was a higher prevalence in patients over 65 years of age. Thus, it remains to be determined whether the loss of tolerance with aging facilitates production of these and other autoantibodies that might worsen clinical outcome in COVID-19. Moreover, the mechanistic basis of the sex bias to produce such autoantibodies specifically in males also warrants further investigation.

\section{Bolstering immunity in older adults to combat COVID-19}

Biomarkers of aging. While we associate immunosenescence with the chronological process of aging, this paradigm can be misleading. People of the same chronological age can have a widely variable 'immunological age'. Important work is being carried out to identify biomarkers associated with immunological aging, in the hope of developing prognostic tools for assessing an individual's risk of developing specific age-related diseases ${ }^{16}$. Identifying biomarkers indicative of immunosenescent phenotypes would also be of great interest for the development of therapeutics that could pre-emptively bolster viral immunity not only in older individuals but also in susceptible younger individuals.

Identifying biomarkers of immunosenescence represents an especially exciting area of research, as the effects of aging have been shown to be amenable to interventions. For example, epigenetic changes associated with aging, including Horvath's methylation clock $^{164}$, appear to be responsive to unique pharmacological interventions. One year of treatment with dehydroepiandrosterone, metformin and recombinant human growth hormone therapy in elderly men resulted in a 1.5 -year reduction in 'epigenetic age' 
and significantly regenerated the thymus ${ }^{165}$. Many strategies have already been proposed to ameliorate the declining immune system in older adults ${ }^{166}$. Below, we will highlight how current strategies to combat aging could be linked to improve immune function in the face of SARS-CoV-2.

Interventions for immunosenescence and COVID-19. Rapamycin and rapalogs. Sirolimus (rapamycin) and rapalogs, derivatives and mimetics of rapamycin, have often been studied in the field of aging. These drugs target critical factors in the rapamycin (TOR) pathway and are commonly used clinically as immunosuppressants. Animal models exploring the effect of rapamycin and rapalogs on longevity have determined their ability to extend lifespan ${ }^{167-169}$. While they are immunosuppressive at high doses, these compounds exert immunostimulatory effects at lower doses ${ }^{170}$. Administration of a rapalog, mTOR inhibitor RAD001, improved response to the influenza vaccine in older individuals by around $20 \%{ }^{171}$.

The mechanism of immunostimulatory action by rapamycin and rapalogs is not quite clear. One possibility is that inhibition of mTORC1 could be relieving feedback inhibition on other inflammatory and metabolic pathways, thus boosting immunity. One such pathway could be insulin signaling. Insulin is a critical mediator of adaptive immune effector function against respiratory infections ${ }^{172}$, and mTORC1 activation of S6 kinase blocks insulin signaling ${ }^{173,174}$. mTOR was also shown to regulate STAT signaling ${ }^{175}$, and studies in older humans show that elevation in baseline phosphorylation of STAT proteins in T cells distinguishes healthy aging individuals from unhealthy aging individuals based on cardiovascular aging phenotypes ${ }^{17}$. However, rapamycin was also observed to enhance the expression of IL-6 (refs. ${ }^{176-178}$ ), which is associated with poor COVID-19 outcomes, and patients with type 2 diabetes and COVID-19 had significantly increased mortality rates when receiving insulin treatment during the course of their hospitalization $^{179}$. Thus, before any treatment or adjuvant uses for rapamycin and rapalogs are considered, further investigation into how mTOR and other nutrient-sensing pathways alter immunosenescence and COVID-19 outcomes are necessary.

Senolytics. Numerous clinical trials are also underway to test the role of senolytic compounds on aging. Senolytics, such as fisetin and quercetin and dasatinib $(D+Q)$, clear senescent cells and reduce the pro-inflammatory and pro-thrombotic effects of the SASP. Several small studies have found an improvement in symptoms of inflammation with the administration of senolytics. For example, $\mathrm{D}+\mathrm{Q}$ resulted in a reduction in SASP-associated pro-inflammatory cytokines during idiopathic pulmonary fibrosis, an age-related lung disease ${ }^{180}$, and a recent study showed that fisetin and D+Q reduced SASP and improved survival in a mouse model of $\beta$-coronavirus ${ }^{84}$. Quercetin may also have beneficial renal-protective and senolyticindependent effects during SARS-CoV-2 infection ${ }^{181}$. However, more work is needed to determine whether senolytics could be a viable option in humans to rejuvenate an aged immune system as either a preventative measure before SARS-CoV-2 infection or as a treatment option during acute infection.

$\mathrm{NAD}^{+}$precursors. Therapeutics targeting $\mathrm{NAD}^{+}$metabolism have also been proposed as potential treatments for age-related immune decline, as reduced $\mathrm{NAD}^{+}$levels are associated with impaired mitochondrial function, immune cell metabolic reprogramming and cell function ${ }^{182}$. A recent paper described the downregulation of nuclear-encoded mitochondrial genes related to cellular respiration and complex I during infection with SARS-CoV-2, suggesting that proper mitochondrial function of immune cells is essential for containing viral propagation ${ }^{183}$. Immune system activation can further reduce $\mathrm{NAD}^{+}$levels, as was recently shown in macrophages treated with PAMPs, including viral TLR ligands, and in aging mouse tissues that contain CD38-dependent NADase activity ${ }^{184}$. Administration of the $\mathrm{NAD}^{+}$precursor nicotinamide mononucleotide in mice has been somewhat promising at restoring $\mathrm{NAD}^{+}$levels and improving mitochondrial function ${ }^{185}$. A similar $\mathrm{NAD}^{+}$precursor, nicotinamide riboside, was also able to restore $\mathrm{NAD}^{+}$levels in mice and humans ${ }^{186}$.

Diet modulation. There is a mutual interaction between nutrition, immune function and inflammatory state, as detailed among the hallmarks of immunosenescence ${ }^{187}$. Notably, a high rate of long-living people and the low incidence of cardiovascular disease in many Mediterranean countries suggests the importance of a diet rich in fruits, vegetables, whole grains, legumes, fish high in omega-3 fatty acids and extra virgin olive oil ${ }^{188}$. This particular diet results in a reduction in both oxidative stress and inflammation and regulation of eubiosis of the gut microbiota, which, in turn, all contribute to an improvement in immune responses ${ }^{189}$. The use of probiotics in older adults was also shown to regulate inflammatory conditions: specifically, probiotics can attenuate the production of both IL-1 $\beta$ and IL-6 (ref. ${ }^{190}$ ), which are associated with severe COVID-19. The Mediterranean diet also modulates the level of many biomarkers of inflammation, including IL-6 (ref. ${ }^{187}$ ).

Another possible dietary approach to the reversion of immunosenescence is caloric restriction. NF- $\kappa \mathrm{B}, \mathrm{mTOR}$, AMP-activated protein kinase (AMPK) and MAPK are pathways that are involved in both aging and inflammation and have been shown to be affected by caloric restriction, resulting in downregulation of inflammatory markers, such as IL-6 and IL-1 $\beta^{191,192}$. Clinical trials using metformin, which activates AMPK, to target aging have already begun ${ }^{193}$. Additionally, ketones, which are largely produced during either ketogenic diets or fasting periods, can impact immune function and may have possible therapeutic effects for COVID-19 (ref. ${ }^{194}$ ). If caloric restriction can reverse the age-related upregulation of inflammatory genes, future studies should compare COVID-19 responses in elderly patients segmented by caloric intake and fasting regimes. Undernutrition, however, is common in older people and is also linked to impaired immune responses ${ }^{187}$. Restricting glucose use can also be fatal in mice during viral infection, even though it proves beneficial for fighting bacterial infections ${ }^{195}$. Therefore, more work is needed to determine whether reduced caloric intake or specific types of nutritional supplementation may boost or hinder immune responses with age in the specific context of SARS-CoV-2 infection.

\section{Conclusions and perspectives}

The serious effects of SARS-CoV-2 infection are likely to be caused by a pathological hyperinflammatory response initiating uncontrolled local tissue damage, vascular leakage, systemic cytokine storm and thrombosis. Research has begun to identify pathophysiological mechanisms underlying these events in response to SARS$\mathrm{CoV}-2$, and some recurring observations in cases of severe disease have emerged: (1) early defects in type I IFN production and signaling, (2) suboptimal T cell responses and (3) dysregulated monocyte and neutrophil inflammation. While there is much work left to elucidate why some develop severe disease while others are asymptomatic, we can begin to propose why an aged immune system may be especially vulnerable to these severe outcomes.

Basal inflammaging is driven by NF- $\mathrm{BB}$ signaling and generally renders immune cells initially hyporesponsive to acute activation, with the possible exception of monocyte subsets ${ }^{71,81}$. A lack of early type I IFN responses during severe COVID-19, compounded with the JAK-STAT hyporesponsiveness of aged immune cells ${ }^{17}$, could facilitate early viral replication and overburden the immune system during early responses to SARS-CoV-2 invasion in older individuals. Once the aged immune system overcomes initial signaling thresholds and local viral load peaks, massive amounts of 
pro-inflammatory cytokines would be released, and tissue destruction and vascular permeability may become tipped too far in favor of propagating continued, hyperinflammatory, pathological innate responses. This 'tipping point' may also be reached through further TLR4 priming due to secondary bacterial superinfections resulting from the initial viral invasion ${ }^{53}$. Meanwhile, poor adaptive responses to SARS-CoV-2 in older individuals could be partly driven by ineffective $\mathrm{T}$ cell priming, a lack of naive $\mathrm{T}$ cell diversity, diminished antibody maturation and/or inefficient pre-existing memory; these characteristics of immunosenescence are likely to be compounded by viral evasion tactics and improper early innate responses. Without the help of a fully functional antigen-specific immune response, the broadly acting innate immune system will continue to drive highly destructive effects of inflammation in an unregulated manner, leading to the enhanced morbidity of severe COVID-19.

SARS-CoV-2 has highlighted gaps in our knowledge of the aged immune system. Even a basic understanding of how specific immune cell subsets react to insults as we age remains a major topic of research. Most studies involve the interrogation of human immune cells taken from the blood, but analysis of human cells from specific tissues is lacking. While mouse studies allow us to glean information on immunosenescence, differences between mouse and human immune aging have already been discovered, such as in mechanisms controlling naive $\mathrm{T}$ cell repertoire diversity $^{135}$. Furthermore, immune cell subsets are not always perfectly conserved across the two species, such as in the case of monocyte subsets $^{196}$. These examples emphasize the need for caution when extrapolating data from one model to the next. Aging immunology studies should also better integrate emerging insights from geroscience, especially from inflammaging research, which has recently described pivotal roles for mitochondrial dysfunction, proteostasis, nutrient sensing and physical changes in the tissue microenvironment in modulating immune pathways ${ }^{197-200}$.

Recent early successes of the Pfizer and Moderna mRNA COVID-19 vaccines appear promising for the elderly population. In stage III clinical trials, the Pfizer vaccine (BNT162b2) had a $94.7 \%$ success rate for those aged 65 years or older (with a 95\% confidence interval of 66.7-99.9\%) ${ }^{201}$, and the Moderna vaccine (mRNA-1273) was $86.4 \%$ efficient in the same age group (with a $95 \%$ confidence interval of 61.4-95.2\%) $)^{202}$. While these preliminary results are exciting, both studies assessed vaccine efficiency for only about $90 \mathrm{~d}$ after complete dosing. A more recent study with BNT162b2 showed that people over the age of 80 had lower anti-SARS-CoV-2 antibody titers $17 \mathrm{~d}$ after the second dose when compared to those under 60 years of age, including $31 \%$ with no detectable neutralizing antibodies $^{203}$. Thus, long-term follow-up studies are needed to determine whether there is sustained efficacy in older individuals. Still, many people have already been infected with SARS-CoV-2, and the longterm effects of this infection have yet to be extensively detailed. Chronic infections, such as with CMV, greatly affect aging phenotypes, and it is possible that COVID-19 may leave a lasting imprint on human physiology as well.

The field of geroscience has been given a boost of attention from the COVID-19 pandemic, which will hopefully lead to even more advances and breakthroughs in the near future. Indeed, our first defense against any virus is a properly functioning immune system; thus, we need to further elucidate mechanisms of immunosenescence and pursue the development of therapeutics that will enhance 'healthspan' in a pre-emptive way.

Received: 16 February 2021; Accepted: 2 August 2021; Published online: 14 September 2021

\section{References}

1. Yoshikawa, T. T. Epidemiology and unique aspects of aging and infectious diseases. Clin. Infect. Dis. 30, 931-933 (2000).
2. Goodwin, K., Viboud, C. \& Simonsen, L. Antibody response to influenza vaccination in the elderly: a quantitative review. Vaccine 24, 1159-1169 (2006)

3. Melegaro, A. \& Edmunds, W. J. The 23-valent pneumococcal polysaccharide vaccine. Part I. Efficacy of PPV in the elderly: a comparison of metaanalyses. Eur. J. Epidemiol. 19, 353-363 (2004).

4. Wolters, B., Junge, U., Dziuba, S. \& Roggendorf, M. Immunogenicity of combined hepatitis A and B vaccine in elderly persons. Vaccine 21, 3623-3628 (2003).

5. Kaml, M. et al. Booster vaccination in the elderly: their success depends on the vaccine type applied earlier in life as well as on pre-vaccination antibody titers. Vaccine 24, 6808-6811 (2006).

6. Hainz, U. et al. Insufficient protection for healthy elderly adults by tetanus and TBE vaccines. Vaccine 23, 3232-3235 (2005).

7. Wu, C. et al. Risk factors associated with acute respiratory distress syndrome and death in patients with coronavirus disease 2019 pneumonia in Wuhan, China. JAMA Intern. Med. 180, 934-943 (2020).

8. Santesmasses, D. et al. COVID-19 is an emergent disease of aging. Aging Cell 19, e13230 (2020).

9. Zheng, Z. et al. Risk factors of critical \& mortal COVID-19 cases: a systematic literature review and meta-analysis. J. Infect. 81, e16-e25 (2020).

10. Bajgain, K. T., Badal, S., Bajgain, B. B. \& Santana, M. J. Prevalence of comorbidities among individuals with COVID-19: a rapid review of current literature. Am. J. Infect. Control 49, 238-246 (2021).

11. Centers for Disease Control and Prevention. COVID Data Tracker. Vol. 2021 (accessed 24 May 2021): https://covid.cdc.gov/covid-data-tracker

12. Gold, J. A. W. et al. Race, ethnicity, and age trends in persons who died from COVID-19-United States, May-August 2020. MMWR Morb. Mortal. Wkly Rep. 69, 1517-1521 (2020).

13. Ho, F. K. et al. Is older age associated with COVID-19 mortality in the absence of other risk factors? General population cohort study of 470,034 participants. PLoS ONE 15, e0241824 (2020).

14. Ferrucci, L. \& Fabbri, E. Inflammageing: chronic inflammation in ageing, cardiovascular disease, and frailty. Nat. Rev. Cardiol. 15, 505-522 (2018).

15. Furman, D. et al. Chronic inflammation in the etiology of disease across the life span. Nat. Med. 25, 1822-1832 (2019).

16. Alpert, A. et al. A clinically meaningful metric of immune age derived from high-dimensional longitudinal monitoring. Nat. Med. 25, 487-495 (2019).

17. Shen-Orr, S. S. et al. Defective signaling in the JAK-STAT pathway tracks with chronic inflammation and cardiovascular risk in aging humans. Cell Syst. 3, 374-384 (2016).

18. Rea, I. M. et al. Age and age-related diseases: role of inflammation triggers and cytokines. Front. Immunol. 9, 586 (2018).

19. Chougnet, C. A. et al. Loss of phagocytic and antigen cross-presenting capacity in aging dendritic cells is associated with mitochondrial dysfunction. J. Immunol. 195, 2624-2632 (2015).

20. Cumberbatch, M., Dearman, R. J. \& Kimber, I. Influence of ageing on Langerhans cell migration in mice: identification of a putative deficiency of epidermal interleukin-1 $\beta$. Immunology 105, 466-477 (2002).

21. Manser, A. R. \& Uhrberg, M. Age-related changes in natural killer cell repertoires: impact on NK cell function and immune surveillance. Cancer Immunol. Immunother. 65, 417-426 (2016).

22. Metcalf, T. U. et al. Global analyses revealed age-related alterations in innate immune responses after stimulation of pathogen recognition receptors. Aging Cell 14, 421-432 (2015).

23. Metcalf, T. U. et al. Human monocyte subsets are transcriptionally and functionally altered in aging in response to pattern recognition receptor agonists. J. Immunol. 199, 1405-1417 (2017).

24. van Duin, D. et al. Age-associated defect in human TLR-1/2 function. J. Immunol. 178, 970-975 (2007)

25. Zacca, E. R. et al. Aging impairs the ability of conventional dendritic cells to cross-prime $\mathrm{CD} 8^{+} \mathrm{T}$ cells upon stimulation with a TLR7 ligand. PLoS ONE 10, e0140672 (2015)

26. Fourati, S. et al. Pre-vaccination inflammation and B-cell signalling predict age-related hyporesponse to hepatitis B vaccination. Nat. Commun. 7, 10369 (2016).

27. Verschoor, C. P. et al. Serum C-reactive protein and congestive heart failure as significant predictors of herpes zoster vaccine response in elderly nursing home residents. J. Infect. Dis. 216, 191-197 (2017).

28. McDade, T. W., Adair, L., Feranil, A. B. \& Kuzawa, C. Positive antibody response to vaccination in adolescence predicts lower $\mathrm{C}$-reactive protein concentration in young adulthood in the Philippines. Am. J. Hum. Biol. 23, 313-318 (2011)

29. Derhovanessian, E., Solana, R., Larbi, A. \& Pawelec, G. Immunity, ageing and cancer. Immun. Ageing 5, 11 (2008).

30. Weiskopf, D., Weinberger, B. \& Grubeck-Loebenstein, B. The aging of the immune system. Transpl. Int. 22, 1041-1050 (2009).

31. Nikolich-Zugich, J. et al. SARS-CoV-2 and COVID-19 in older adults: what we may expect regarding pathogenesis, immune responses, and outcomes. GeroScience 42, 505-514 (2020). 
32. Nikolich-Zugich, J. The twilight of immunity: emerging concepts in aging of the immune system. Nat. Immunol. 19, 10-19 (2018).

33. Sadighi Akha, A. A. Aging and the immune system: an overview. J. Immunol. Methods 463, 21-26 (2018).

34. Boraschi, D. \& Italiani, P. Immunosenescence and vaccine failure in the elderly: strategies for improving response. Immunol. Lett. 162, 346-353 (2014).

35. Zhou, P. et al. A pneumonia outbreak associated with a new coronavirus of probable bat origin. Nature 579, 270-273 (2020).

36. Li, F. Structure, function, and evolution of coronavirus spike proteins. Annu. Rev. Virol. 3, 237-261 (2016).

37. Wang, Q. et al. Structural and functional basis of SARS-CoV-2 entry by using human ACE2. Cell 181, 894-904 (2020).

38. Hoffmann, M. et al. SARS-CoV-2 cell entry depends on ACE2 and TMPRSS2 and is blocked by a clinically proven protease inhibitor. Cell 181, 271-280 (2020).

39. Sungnak, W. et al. SARS-CoV-2 entry factors are highly expressed in nasal epithelial cells together with innate immune genes. Nat. Med. 26, 681-687 (2020).

40. Khanmohammadi, S. \& Rezaei, N. Role of Toll-like receptors in the pathogenesis of COVID-19. J. Med. Virol. 93, 2735-2739 (2021).

41. Merad, M. \& Martin, J. C. Pathological inflammation in patients with COVID-19: a key role for monocytes and macrophages. Nat. Rev. Immunol. 20, 355-362 (2020).

42. Onofrio, L. et al. Toll-like receptors and COVID-19: a two-faced story with an exciting ending. Future Sci. OA 6, FSO605 (2020).

43. Chakraborty, C., Sharma, A. R., Sharma, G., Bhattacharya, M. \& Lee, S. S. SARS-CoV-2 causing pneumonia-associated respiratory disorder (COVID-19): diagnostic and proposed therapeutic options. Eur. Rev. Med. Pharmacol. Sci. 24, 4016-4026 (2020).

44. Zhou, F. et al. Clinical course and risk factors for mortality of adult inpatients with COVID-19 in Wuhan, China: a retrospective cohort study. Lancet 395, 1054-1062 (2020).

45. Huang, C. et al. Clinical features of patients infected with 2019 novel coronavirus in Wuhan, China. Lancet 395, 497-506 (2020).

46. Iba, T., Levy, J. H., Raj, A. \& Warkentin, T. E. Advance in the management of sepsis-induced coagulopathy and disseminated intravascular coagulation. J. Clin. Med. 8, 728 (2019).

47. Simmons, J. \& Pittet, J. F. The coagulopathy of acute sepsis. Curr. Opin. Anaesthesiol. 28, 227-236 (2015).

48. Papazian, L. et al. Diagnostic workup for ARDS patients. Intensive Care Med. 42, 674-685 (2016).

49. Xu, Z. et al. Pathological findings of COVID-19 associated with acute respiratory distress syndrome. Lancet Respir. Med. 8, 420-422 (2020).

50. Desai, N. et al. Temporal and spatial heterogeneity of host response to SARS-CoV-2 pulmonary infection. Nat. Commun. 11, 6319 (2020).

51. Ruan, Q., Yang, K., Wang, W., Jiang, L. \& Song, J. Clinical predictors of mortality due to COVID-19 based on an analysis of data of 150 patients from Wuhan, China. Intensive Care Med. 46, 846-848 (2020).

52. Vaillancourt, M. \& Jorth, P. The unrecognized threat of secondary bacterial infections with COVID-19. mBio 11, e01806-20 (2020)

53. Wang, A. et al. Specific sequences of infectious challenge lead to secondary hemophagocytic lymphohistiocytosis-like disease in mice. Proc. Natl Acad. Sci. USA 116, 2200-2209 (2019).

54. Dinnon, K. H. III et al. A mouse-adapted model of SARS-CoV-2 to test COVID-19 countermeasures. Nature 586, 560-566 (2020).

55. Leist, S. R. et al. A mouse-adapted SARS-CoV-2 induces acute lung injury and mortality in standard laboratory mice. Cell 183, 1070-1085 (2020).

56. Garcia, L. F. Immune response, inflammation, and the clinical spectrum of COVID-19. Front. Immunol. 11, 1441 (2020).

57. Torres Acosta, M. A. \& Singer, B. D. Pathogenesis of COVID-19-induced ARDS: implications for an ageing population. Eur. Respir. J. 56, 2002049 (2020).

58. Tay, M. Z., Poh, C. M., Renia, L., MacAry, P. A. \& Ng, L. F. P. The trinity of COVID-19: immunity, inflammation and intervention. Nat. Rev. Immunol. 20, 363-374 (2020).

59. Wang, M., Jiang, L., Monticone, R. E. \& Lakatta, E. G. Proinflammation: the key to arterial aging. Trends Endocrinol. Metab. 25, 72-79 (2014).

60. Imai, Y. et al. Angiotensin-converting enzyme 2 protects from severe acute lung failure. Nature 436, 112-116 (2005)

61. Yu, X. et al. Angiotensin-converting enzyme 2-angiotensin (1-7)-Mas axis prevents pancreatic acinar cell inflammatory response via inhibition of the p38 mitogen-activated protein kinase/nuclear factor-кB pathway. Int. J. Mol. Med. 41, 409-420 (2018).

62. Banu, N., Panikar, S. S., Leal, L. R. \& Leal, A. R. Protective role of ACE2 and its downregulation in SARS-CoV-2 infection leading to macrophage activation syndrome: therapeutic implications. Life Sci. 256, 117905 (2020).

63. Xie, X., Chen, J., Wang, X., Zhang, F. \& Liu, Y. Age- and gender-related difference of ACE2 expression in rat lung. Life Sci. 78, 2166-2171 (2006).
64. Chen, J. et al. Individual variation of the SARS-CoV-2 receptor ACE2 gene expression and regulation. Aging Cell 19, e13168 (2020).

65. Tikellis, C. \& Thomas, M. C. Angiotensin-converting enzyme 2 (ACE2) is a key modulator of the renin angiotensin system in health and disease. Int. J. Pept. 2012, 256294 (2012).

66. Liu, Y. et al. Clinical and biochemical indexes from 2019-nCoV infected patients linked to viral loads and lung injury. Sci. China Life Sci. 63, 364-374 (2020).

67. Rosa, B. A. et al. IFN signaling and neutrophil degranulation transcriptional signatures are induced during SARS-CoV-2 infection. Commun. Biol. 4, 290 (2021)

68. Qin, C. et al. Dysregulation of immune response in patients with coronavirus 2019 (COVID-19) in Wuhan, China. Clin. Infect. Dis. 71, 762-768 (2020).

69. Johnson, T. E. Recent results: biomarkers of aging. Exp. Gerontol. 41, 1243-1246 (2006)

70. Brasier, A. R. The nuclear factor- $\kappa \mathrm{B}-$ interleukin- 6 signalling pathway mediating vascular inflammation. Cardiovasc. Res. 86, 211-218 (2010).

71. Salminen, A. et al. Activation of innate immunity system during aging: NF-kB signaling is the molecular culprit of inflamm-aging. Ageing Res. Rev. 7, 83-105 (2008)

72. Guaraldi, G. et al. Tocilizumab in patients with severe COVID-19: a retrospective cohort study. Lancet Rheumatol. 2, e474-e484 (2020).

73. Youm, Y. H. et al. Canonical Nlrp3 inflammasome links systemic low-grade inflammation to functional decline in aging. Cell Metab. 18, 519-532 (2013).

74. Junqueira, C. et al. SARS-CoV-2 infects blood monocytes to activate NLRP3 and AIM2 inflammasomes, pyroptosis and cytokine release. Preprint at medRxiv https://doi.org/10.1101/2021.03.06.21252796 (2021).

75. Rodrigues, T. S. et al. Inflammasomes are activated in response to SARS-CoV-2 infection and are associated with COVID-19 severity in patients. J. Exp. Med. 218, e20201707 (2021).

76. Zheng, Y. et al. A human circulating immune cell landscape in aging and COVID-19. Protein Cell 11, 740-770 (2020).

77. Mishra, S. R. et al. Mitochondrial dysfunction as a driver of NLRP3 inflammasome activation and its modulation through mitophagy for potential therapeutics. Int. J. Biochem. Cell Biol. 136, 106013 (2021).

78. Xian, H. et al. Metformin inhibition of mitochondrial ATP and DNA synthesis abrogates NLRP3 inflammasome activation and pulmonary inflammation. Immunity 54, 1463-1477 (2021).

79. Barkas, F. et al. Anakinra in hospitalized non-intubated patients with coronavirus disease 2019: a systematic review and meta-analysis. Rheumatology https://doi.org/10.1093/rheumatology/keab447 (2021).

80. Shah, A. Novel coronavirus-induced NLRP3 inflammasome activation: a potential drug target in the treatment of COVID-19. Front. Immunol. 11, 1021 (2020).

81. Sayed, N. et al. An inflammatory aging clock (iAge) based on deep learning tracks multi-morbidity, immunosenescence, frailty and cardiovascular aging. Nat. Aging 1, 598-615 (2021).

82. Krishnamurthy, J. et al. Ink4a/Arf expression is a biomarker of aging. J. Clin. Invest. 114, 1299-1307 (2004).

83. Coppe, J. P., Desprez, P. Y., Krtolica, A. \& Campisi, J. The senescenceassociated secretory phenotype: the dark side of tumor suppression. Annu Rev. Pathol. 5, 99-118 (2010).

84. Camell, C. D. et al. Senolytics reduce coronavirus-related mortality in old mice. Science 373, eabe4832 (2021).

85. Ortmann, W. \& Kolaczkowska, E. Age is the work of art? Impact of neutrophil and organism age on neutrophil extracellular trap formation. Cell Tissue Res. 371, 473-488 (2018)

86. Sapey, E. et al. Phosphoinositide 3-kinase inhibition restores neutrophil accuracy in the elderly: toward targeted treatments for immunosenescence. Blood 123, 239-248 (2014).

87. Asensi, V. et al. In vivo interleukin-6 protects neutrophils from apoptosis in osteomyelitis. Infect. Immun. 72, 3823-3828 (2004)

88. Liu, Y. et al. Neutrophil-to-lymphocyte ratio as an independent risk factor for mortality in hospitalized patients with COVID-19. J. Infect. 81, e6-e12 (2020).

89. Kuri-Cervantes, L. et al. Comprehensive mapping of immune perturbations associated with severe COVID-19. Sci. Immunol. 5, eabd7114 (2020).

90. Lucas, C. et al. Longitudinal analyses reveal immunological misfiring in severe COVID-19. Nature 584, 463-469 (2020).

91. Bajaj, V. et al. Aging, immunity, and COVID-19: how age influences the host immune response to coronavirus infections? Front. Physiol. 11, 571416 (2020).

92. Wiley, C. D. et al. SILAC analysis reveals increased secretion of hemostasisrelated factors by senescent cells. Cell Rep. 28, 3329-3337 (2019).

93. Tang, N., Li, D., Wang, X. \& Sun, Z. Abnormal coagulation parameters are associated with poor prognosis in patients with novel coronavirus pneumonia. J. Thromb. Haemost. 18, 844-847 (2020). 
94. Guan, W. J. et al. Clinical characteristics of coronavirus disease 2019 in China. N. Engl. J. Med. 382, 1708-1720 (2020).

95. Chen, N. et al. Epidemiological and clinical characteristics of 99 cases of 2019 novel coronavirus pneumonia in Wuhan, China: a descriptive study. Lancet 395, 507-513 (2020).

96. Cui, S., Chen, S., Li, X., Liu, S. \& Wang, F. Prevalence of venous thromboembolism in patients with severe novel coronavirus pneumonia. J. Thromb. Haemost. 18, 1421-1424 (2020).

97. Klok, F. A. et al. Incidence of thrombotic complications in critically ill ICU patients with COVID-19. Thromb. Res. 191, 145-147 (2020).

98. Lodigiani, C. et al. Venous and arterial thromboembolic complications in COVID-19 patients admitted to an academic hospital in Milan, Italy. Thromb. Res. 191, 9-14 (2020).

99. Fox, S. E. et al. Pulmonary and cardiac pathology in African American patients with COVID-19: an autopsy series from New Orleans. Lancet Respir. Med. 8, 681-686 (2020).

100. Price, L. C., McCabe, C., Garfield, B. \& Wort, S. J. Thrombosis and COVID-19 pneumonia: the clot thickens! Eur. Respir. J. 56, 2001608 (2020).

101. Zuo, Y. et al. Prothrombotic autoantibodies in serum from patients hospitalized with COVID-19. Sci. Transl. Med. 12, eabd3876 (2020)

102. Hadjadj, J. et al. Impaired type I interferon activity and inflammatory responses in severe COVID-19 patients. Science 369, 718-724 (2020).

103. Zhou, R. et al. Acute SARS-CoV-2 infection impairs dendritic cell and $\mathrm{T}$ cell responses. Immunity 53, 864-877 (2020).

104. Bastard, P. et al. Autoantibodies against type I IFNs in patients with life-threatening COVID-19. Science 370, eabd4585 (2020).

105. Zhang, Q. et al. Inborn errors of type I IFN immunity in patients with life-threatening COVID-19. Science 370, eabd4570 (2020).

106. McNab, F., Mayer-Barber, K., Sher, A., Wack, A. \& O'Garra, A. Type I interferons in infectious disease. Nat. Rev. Immunol. 15, 87-103 (2015).

107. Channappanavar, R. et al. Dysregulated type I interferon and inflammatory monocyte-macrophage responses cause lethal pneumonia in SARS-CoVinfected mice. Cell Host Microbe 19, 181-193 (2016).

108. Channappanavar, R. et al. IFN-I response timing relative to virus replication determines MERS coronavirus infection outcomes. J. Clin. Invest. 129, 3625-3639 (2019).

109. Silvin, A. et al. Elevated calprotectin and abnormal myeloid cell subsets discriminate severe from mild COVID-19. Cell 182, 1401-1418 (2020).

110. Schulte-Schrepping, J. et al. Severe COVID-19 is marked by a dysregulated myeloid cell compartment. Cell 182, 1419-1440 (2020).

111. Wong, K. L. et al. The three human monocyte subsets: implications for health and disease. Immunol. Res. 53, 41-57 (2012).

112. Zhou, Y. et al. Pathogenic T-cells and inflammatory monocytes incite inflammatory storms in severe COVID-19 patients. Natl Sci. Rev. 7, 998-1002 (2020)

113. Zhang, D. et al. COVID-19 infection induces readily detectable morphologic and inflammation-related phenotypic changes in peripheral blood monocytes. J. Leuk. Biol. 109, 13-22 (2021).

114. Boyette, L. B. et al. Phenotype, function, and differentiation potential of human monocyte subsets. PLoS ONE 12, e0176460 (2017).

115. Molony, R. D. et al. Aging impairs both primary and secondary RIG-I signaling for interferon induction in human monocytes. Sci. Signal. 10, eaan2392 (2017)

116. Shi, C. S. et al. SARS-coronavirus open reading frame-9b suppresses innate immunity by targeting mitochondria and the MAVS/TRAF3/TRAF6 signalosome. J. Immunol. 193, 3080-3089 (2014).

117. Feng, E., Balint, E., Poznanski, S. M., Ashkar, A. A. \& Loeb, M. Aging and interferons: impacts on inflammation and viral disease outcomes. Cells 10, eaan2392 (2021).

118. Yamada, T. et al. RIG-I triggers a signaling-abortive anti-SARS-CoV-2 defense in human lung cells. Nat. Immunol. 22, 820-828 (2021).

119. Shodell, M. \& Siegal, F. P. Circulating, interferon-producing plasmacytoid dendritic cells decline during human ageing. Scand. J. Immunol. 56, 518-521 (2002).

120. Perez-Cabezas, B. et al. Reduced numbers of plasmacytoid dendritic cells in aged blood donors. Exp. Gerontol. 42, 1033-1038 (2007).

121. Jing, Y. et al. Aging is associated with a numerical and functional decline in plasmacytoid dendritic cells, whereas myeloid dendritic cells are relatively unaltered in human peripheral blood. Hum. Immunol. 70, 777-784 (2009)

122. Giamarellos-Bourboulis, E. J. et al. Complex immune dysregulation in COVID-19 patients with severe respiratory failure. Cell Host Microbe 27, 992-1000 (2020)

123. Seidler, S., Zimmermann, H. W., Bartneck, M., Trautwein, C. \& Tacke, F. Age-dependent alterations of monocyte subsets and monocyte-related chemokine pathways in healthy adults. BMC Immunol. 11, 30 (2010).

124. Wong, C. \& Goldstein, D. R. Impact of aging on antigen presentation cell function of dendritic cells. Curr. Opin. Immunol. 25, 535-541 (2013).

125. Zhao, J., Zhao, J., Legge, K. \& Perlman, S. Age-related increases in PGD expression impair respiratory DC migration, resulting in diminished $\mathrm{T}$ cell responses upon respiratory virus infection in mice. J. Clin. Invest. 121, 4921-4930 (2011)

126. Rydyznski Moderbacher, C. et al. Antigen-specific adaptive immunity to SARS-CoV-2 in acute COVID-19 and associations with age and disease severity. Cell 183, 996-1012 (2020)

127. Grifoni, A. et al. Targets of T cell responses to SARS-CoV-2 coronavirus in humans with COVID-19 disease and unexposed individuals. Cell 181, 1489-1501 (2020).

128. Braun, J. et al. SARS-CoV-2-reactive T cells in healthy donors and patients with COVID-19. Nature 587, 270-274 (2020).

129. Kaneko, N. et al. Loss of Bcl-6-expressing T follicular helper cells and germinal centers in COVID-19. Cell 183, 143-157 (2020).

130. Zhao, J., Zhao, J. \& Perlman, S. T cell responses are required for protection from clinical disease and for virus clearance in severe acute respiratory syndrome coronavirus-infected mice. J. Virol. 84, 9318-9325 (2010).

131. Nelde, A. et al. SARS-CoV-2-derived peptides define heterologous and COVID-19-induced T cell recognition. Nat. Immunol. 22, 74-85 (2021).

132. Peng, $\mathrm{Y}$. et al. Broad and strong memory $\mathrm{CD} 4^{+}$and $\mathrm{CD} 8^{+} \mathrm{T}$ cells induced by SARS-CoV-2 in UK convalescent individuals following COVID-19. Nat. Immunol. 21, 1336-1345 (2020).

133. Britanova, O. V. et al. Age-related decrease in TCR repertoire diversity measured with deep and normalized sequence profiling. J. Immunol. 192, 2689-2698 (2014).

134. Gruver, A. L., Hudson, L. L. \& Sempowski, G. D. Immunosenescence of ageing. J. Pathol. 211, 144-156 (2007).

135. Goronzy, J. J. \& Weyand, C. M. Mechanisms underlying T cell ageing. Nat. Rev. Immunol. 19, 573-583 (2019)

136. Huang, I. \& Pranata, R. Lymphopenia in severe coronavirus disease-2019 (COVID-19): systematic review and meta-analysis. J. Intensive Care $\mathbf{8}$, 36 (2020).

137. Tan, L. et al. Lymphopenia predicts disease severity of COVID-19: a descriptive and predictive study. Signal Transduct. Target. Ther. 5, 33 (2020).

138. Liao, M. et al. Single-cell landscape of bronchoalveolar immune cells in patients with COVID-19. Nat. Med. 26, 842-844 (2020).

139. Diao, B. et al. Reduction and functional exhaustion of $\mathrm{T}$ cells in patients with coronavirus disease 2019 (COVID-19). Front. Immunol. 11, 827 (2020).

140. Wan, S. et al. Characteristics of lymphocyte subsets and cytokines in peripheral blood of 123 hospitalized patients with 2019 novel coronavirus pneumonia (NCP). Preprint at medRxiv https://doi. org/10.1101/2020.02.10.20021832 (2020).

141. Zheng, H. Y. et al. Elevated exhaustion levels and reduced functional diversity of $\mathrm{T}$ cells in peripheral blood may predict severe progression in COVID-19 patients. Cell. Mol. Immunol. 17, 541-543 (2020).

142. Mazzoni, A. et al. Impaired immune cell cytotoxicity in severe COVID-19 is IL-6 dependent. J. Clin. Invest. 130, 4694-4703 (2020).

143. Rha, M. S. et al. PD-1-expressing SARS-CoV-2-specific $\mathrm{CD}^{+} \mathrm{T}$ cells are not exhausted, but functional in patients with COVID-19. Immunity 54, 44-52 (2021).

144. Mateus, J. et al. Selective and cross-reactive SARS-CoV-2 T cell epitopes in unexposed humans. Science 370, 89-94 (2020).

145. Klenerman, P. \& Oxenius, A. T cell responses to cytomegalovirus. Nat. Rev Immunol. 16, 367-377 (2016).

146. Shrock, E. et al. Viral epitope profiling of COVID-19 patients reveals cross-reactivity and correlates of severity. Science 370, eabd4250 (2020).

147. White, J. T. et al. Virtual memory T cells develop and mediate bystander protective immunity in an IL-15-dependent manner. Nat. Commun. 7, 11291 (2016).

148. Kim, T. S. \& Shin, E. C. The activation of bystander CD ${ }^{+}$T cells and their roles in viral infection. Exp. Mol. Med. 51, 1-9 (2019).

149. Alsoussi, W. B. et al. A potently neutralizing antibody protects mice against SARS-CoV-2 infection. J. Immunol. 205, 915-922 (2020).

150. Zost, S. J. et al. Potently neutralizing and protective human antibodies against SARS-CoV-2. Nature 584, 443-449 (2020).

151. Devarasetti, P. K., Rajasekhar, L., Baisya, R., Sreejitha, K. S. \& Vardhan, Y. K. A review of COVID-19 convalescent plasma use in COVID-19 with focus on proof of efficacy. Immunol. Res. 69, 18-25 (2021).

152. Arvin, A. M. et al. A perspective on potential antibody-dependent enhancement of SARS-CoV-2. Nature 584, 353-363 (2020).

153. Larsen, M. D. et al. Afucosylated IgG characterizes enveloped viral responses and correlates with COVID-19 severity. Science 371, eabc8378 (2020).

154. Frasca, D., Diaz, A., Romero, M. \& Blomberg, B. B. Human peripheral late/ exhausted memory B cells express a senescent-associated secretory phenotype and preferentially utilize metabolic signaling pathways. Exp. Gerontol. 87, 113-120 (2017).

155. Singh, D. K. et al. Responses to acute infection with SARS-CoV-2 in the lungs of rhesus macaques, baboons and marmosets. Nat. Microbiol. 6, 73-86 (2021). 
156. Cancro, M. P. Age-associated B cells. Annu. Rev. Immunol. 38, 315-340 (2020).

157. Frasca, D. Senescent B cells in aging and age-related diseases: their role in the regulation of antibody responses. Exp. Gerontol. 107, 55-58 (2018).

158. Woodruff, M. C. et al. Extrafollicular B cell responses correlate with neutralizing antibodies and morbidity in COVID-19. Nat. Immunol. 21, 1506-1516 (2020).

159. Duan, Y. Q. et al. Deficiency of $\mathrm{T}_{\mathrm{FH}}$ cells and germinal center in deceased COVID-19 patients. Curr. Med. Sci. 40, 618-624 (2020).

160. Popescu, M., Cabrera-Martinez, B. \& Winslow, G. M. TNF- $\alpha$ contributes to lymphoid tissue disorganization and germinal center B cell suppression during intracellular bacterial infection. J. Immunol. 203, 2415-2424 (2019).

161. Fagiolo, U. et al. Increased cytokine production in mononuclear cells of healthy elderly people. Eur. J. Immunol. 23, 2375-2378 (1993).

162. Bruunsgaard, H., Andersen-Ranberg, K., Hjelmborg, J., Pedersen, B. K. \& Jeune, B. Elevated levels of tumor necrosis factor $\alpha$ and mortality in centenarians. Am. J. Med. 115, 278-283 (2003).

163. Penninx, B. W. et al. Inflammatory markers and incident mobility limitation in the elderly. J. Am. Geriatr. Soc. 52, 1105-1113 (2004).

164. Horvath, S. \& Raj, K. DNA methylation-based biomarkers and the epigenetic clock theory of ageing. Nat. Rev. Genet. 19, 371-384 (2018).

165. Fahy, G. M. et al. Reversal of epigenetic aging and immunosenescent trends in humans. Aging Cell 18, e13028 (2019).

166. Aspinall, R. \& Lang, P. O. Interventions to restore appropriate immune function in the elderly. Immun. Ageing 15, 5 (2018).

167. Kaeberlein, M. et al. Regulation of yeast replicative life span by TOR and Sch9 in response to nutrients. Science 310, 1193-1196 (2005).

168. Robida-Stubbs, S. et al. TOR signaling and rapamycin influence longevity by regulating SKN-1/Nrf and DAF-16/FoxO. Cell Metab. 15, 713-724 (2012).

169. Harrison, D. E. et al. Rapamycin fed late in life extends lifespan in genetically heterogeneous mice. Nature 460, 392-395 (2009).

170. Ferrer, I. R., Araki, K. \& Ford, M. L. Paradoxical aspects of rapamycin immunobiology in transplantation. Am. J. Transplant. 11, 654-659 (2011).

171. Mannick, J. B. et al. mTOR inhibition improves immune function in the elderly. Sci. Transl. Med. 6, 268ra179 (2014).

172. Tsai, S. et al. Insulin receptor-mediated stimulation boosts $\mathrm{T}$ cell immunity during inflammation and infection. Cell Metab. 28, 922-934 (2018).

173. Tremblay, F., Jacques, H. \& Marette, A. Modulation of insulin action by dietary proteins and amino acids: role of the mammalian target of rapamycin nutrient sensing pathway. Curr. Opin. Clin. Nutr. Metab. Care 8, 457-462 (2005).

174. Um, S. H., D’Alessio, D. \& Thomas, G. Nutrient overload, insulin resistance, and ribosomal protein S6 kinase 1, S6K1. Cell Metab. 3, 393-402 (2006).

175. Saleiro, D. \& Platanias, L. C. Intersection of mTOR and STAT signaling in immunity. Trends Immunol. 36, 21-29 (2015).

176. Cappoli, N. et al. The mTOR kinase inhibitor rapamycin enhances the expression and release of pro-inflammatory cytokine interleukin 6 modulating the activation of human microglial cells. EXCLI J. 18, 779-798 (2019).

177. Zhang, Y. et al. Rapamycin upregulates glutamate transporter and IL-6 expression in astrocytes in a mouse model of Parkinson's disease. Cell Death Dis. 8, e2611 (2017)

178. Weichhart, T. et al. The TSC-mTOR signaling pathway regulates the innate inflammatory response. Immunity 29, 565-577 (2008)

179. Yu, B., Li, C., Sun, Y. \& Wang, D. W. Insulin treatment is associated with increased mortality in patients with COVID-19 and type 2 diabetes. Cell Metab. 33, 65-77 (2021).

180. Justice, J. N. et al. Senolytics in idiopathic pulmonary fibrosis: results from a first-in-human, open-label, pilot study. EBioMedicine 40, 554-563 (2019).

181. Gu, Y. Y. et al. Quercetin as a potential treatment for COVID-19-induced acute kidney injury: based on network pharmacology and molecular docking study. PLOS ONE 16, e0245209 (2021).

182. Covarrubias, A. J., Perrone, R., Grozio, A. \& Verdin, E. NAD ${ }^{+}$metabolism and its roles in cellular processes during ageing. Nat. Rev. Mol. Cell Biol. 22 119-141 (2021).

183. Miller, B. et al. Host mitochondrial transcriptome response to SARS-CoV-2 in multiple cell models and clinical samples. Sci. Rep. 11, 3 (2021).

184. Covarrubias, A. J. et al. Senescent cells promote tissue $\mathrm{NAD}^{+}$decline during ageing via the activation of $\mathrm{CD}^{+} 8^{+}$macrophages. Nat. Metab. 2, 1265-1283 (2020).
185. Kiss, T. et al. Nicotinamide mononucleotide (NMN) supplementation promotes neurovascular rejuvenation in aged mice: transcriptional footprint of SIRT1 activation, mitochondrial protection, anti-inflammatory, and anti-apoptotic effects. GeroScience 42, 527-546 (2020).

186. Trammell, S. A. et al. Nicotinamide riboside is uniquely and orally bioavailable in mice and humans. Nat. Commun. 7, 12948 (2016).

187. Aiello, A. et al. Immunosenescence and its hallmarks: how to oppose aging strategically? A review of potential options for therapeutic intervention. Front. Immunol. 10, 2247 (2019).

188. O’Keefe, J. H. et al. A Pesco-Mediterranean diet with intermittent fasting: JACC review topic of the week. J. Am. Coll. Cardiol. 76, 1484-1493 (2020).

189. Mitsou, E. K. et al. Adherence to the Mediterranean diet is associated with the gut microbiota pattern and gastrointestinal characteristics in an adult population. Br. J. Nutr. 117, 1645-1655 (2017).

190. Landete, J. M. et al. Probiotic bacteria for healthier aging: immunomodulation and metabolism of phytoestrogens. Biomed. Res. Int. 2017, 5939818 (2017).

191. Chung, K. W. et al. Recent advances in calorie restriction research on aging. Exp. Gerontol. 48, 1049-1053 (2013).

192. Chung, H. Y., Kim, H. J., Kim, J. W. \& Yu, B. P. The inflammation hypothesis of aging: molecular modulation by calorie restriction. Ann. NY Acad. Sci. 928, 327-335 (2001).

193. Justice, J. N. et al. Development of clinical trials to extend healthy lifespan. Cardiovasc. Endocrinol. Metab. 7, 80-83 (2018).

194. Stubbs, B. J. et al. Investigating ketone bodies as immunometabolic countermeasures against respiratory viral infections. Med 1, 43-65 (2020).

195. Wang, A. et al. Opposing effects of fasting metabolism on tissue tolerance in bacterial and viral inflammation. Cell 166, 1512-1525 (2016).

196. Ziegler-Heitbrock, L. Reprint of: Monocyte subsets in man and other species. Cell. Immunol. 291, 11-15 (2014).

197. Thompson, H. L. et al. Lymph nodes as barriers to T-cell rejuvenation in aging mice and nonhuman primates. Aging Cell 18, e12865 (2019).

198. Chakraborty, M. et al. Mechanical stiffness controls dendritic cell metabolism and function. Cell Rep. 34, 108609 (2021).

199. Desdin-Mico, G. et al. T cells with dysfunctional mitochondria induce multimorbidity and premature senescence. Science 368, 1371-1376 (2020).

200. Bharath, L. P. et al. Metformin enhances autophagy and normalizes mitochondrial function to alleviate aging-associated inflammation. Cell Metab. 32, 44-55 (2020)

201. Polack, F. P. et al. Safety and efficacy of the BNT162b2 mRNA COVID-19 vaccine. N. Engl. J. Med. 383, 2603-2615 (2020).

202. Baden, L. R. et al. Efficacy and safety of the mRNA-1273 SARS-CoV-2 vaccine. N. Engl. J. Med. 384, 403-416 (2020).

203. Muller, L. et al. Age-dependent immune response to the BioNTech/Pfizer BNT162b2 COVID-19 vaccination. Clin. Infect. Dis. https://doi.org/10.1093/ cid/ciab381 (2021).

\section{Acknowledgements}

J.M.B. is a recipient of a National Institutes of Health T32 (AG000266) postdoctoral fellowship award. D.A.W. and D.F. were funded in part through the Impact Circle at the Buck Institute for Research on Aging. Drafts of Figs. 1 and 2 were created with BioRender (A.J.C.).

\section{Author contributions}

All authors contributed to the design and writing of the manuscript and generation of figures.

\section{Competing interests}

J.M.B., D.F., A.J.C., E.V. and D.A.W. declare no competing interests related to this study. D.R. is a chief science officer at Hooke by Healthy, Longevity Optimisation, a longevity clinic and research center.

\section{Additional information}

Correspondence should be addressed to Daniel A. Winer or Eric Verdin.

Reprints and permissions information is available at www.nature.com/reprints.

Publisher's note Springer Nature remains neutral with regard to jurisdictional claims in published maps and institutional affiliations.

(c) Springer Nature America, Inc. 2021 